\title{
Secreted nuclear protein DEK regulates hematopoiesis through CXCR2 signaling
}

\author{
Maegan L. Capitano, ${ }^{1}$ Nirit Mor-Vaknin, ${ }^{2}$ Anjan K. Saha, ${ }^{2}$ Scott Cooper, ${ }^{1}$ Maureen Legendre, ${ }^{2}$ Haihong Guo, ${ }^{3}$ \\ Rafael Contreras-Galindo, ${ }^{2}$ Ferdinand Kappes, ${ }^{3,4}$ Maureen A. Sartor, ${ }^{5,6}$ Christopher T. Lee, ${ }^{6}$ Xinxin Huang, ${ }^{1}$ \\ David M. Markovitz, ${ }^{2}$ and Hal E. Broxmeyer ${ }^{1}$
}

\begin{abstract}
'Department of Microbiology and Immunology, Indiana University School of Medicine, Indianapolis, Indiana, USA. ²Department of Internal Medicine, Division of Infectious Disease, University of Michigan, Ann Arbor, Michigan, USA. ${ }^{3}$ Institute of Biochemistry and Molecular Biology, Medical School, RWTH Aachen University, Aachen, Germany. ${ }^{4}$ Department of Biological Sciences, Xi'an Jiaotong-Liverpool University, Suzhou, China. ${ }^{5}$ Department of Computational Medicine and Bioinformatics, University of Michigan, Ann Arbor, Michigan, USA. ${ }^{6}$ Department of Biostatistics, University of Michigan, Ann Arbor, Michigan, USA.
\end{abstract}

\begin{abstract}
The nuclear protein DEK is an endogenous DNA-binding chromatin factor regulating hematopoiesis. DEK is one of only 2 known secreted nuclear chromatin factors, but whether and how extracellular DEK regulates hematopoiesis is not known. We demonstrated that extracellular DEK greatly enhanced ex vivo expansion of cytokine-stimulated human and mouse hematopoietic stem cells (HSCs) and regulated HSC and hematopoietic progenitor cell (HPC) numbers in vivo and in vitro as determined both phenotypically (by flow cytometry) and functionally (through transplantation and colony formation assays). Recombinant DEK increased long-term HSC numbers and decreased HPC numbers through a mechanism mediated by the CXC chemokine receptor CXCR2 and heparan sulfate proteoglycans (HSPGs) (as determined utilizing CXcr2 ${ }^{-/-}$mice, blocking CXCR2 antibodies, and 3 different HSPC inhibitors) that was associated with enhanced phosphorylation of ERK1/2, AKT, and p38 MAPK. To determine whether extracellular DEK required nuclear function to regulate hematopoiesis, we utilized 2 mutant forms of DEK: one that lacked its nuclear translocation signal and one that lacked DNA-binding ability. Both altered HSC and HPC numbers in vivo or in vitro, suggesting the nuclear function of DEK is not required. Thus, DEK acts as a hematopoietic cytokine, with the potential for clinical applicability.
\end{abstract}

\section{Introduction}

DEK, a conserved, structurally unique DNA-modulating nuclear protein, is involved in global heterochromatin integrity, transcription, DNA replication, and DNA repair, and is implicated in gene regulation (1-3). Interestingly, DEK is secreted by activated human monocyte-derived macrophages and neutrophils in exosome and free forms $(4,5)$. Poly(ADP-ribosyl)ated, hyperphosphorylated DEK is released from T lymphocytes by Fas ligand or stress-mediated apoptosis (6). Secreted DEK chemoattracts neutrophils, cytotoxic T lymphocytes, and natural killer cells through an unidentified receptor, and its secretion is associated with autoimmune diseases $(4,5,7,8)$. DEK is one of two known nuclear proteins that can be secreted with paracrine activity. The other, high-mobility group box 1 (HMGB1) protein, is secreted/released by macrophages under inflammatory conditions $(9-11)$. Secreted

\section{Related Commentary: p. 2205}

Conflict of interest: HEB was previously a member of the Medical Scientific Advisory Board of CORD:USE, a cord blood banking company based in Orlando, Florida, USA, during some of these studies. The anti-DEK aptamers are being patented (patent number 10138486) by the University of Michigan by DMM, NMV, and ML. Copyright: @ 2019, American Society for Clinical Investigation. Submitted: January 15, 2019; Accepted: March 19, 2019; Published: May 20, 2019. Reference information: J Clin Invest. 2019;129(6):2555-2570. https://doi.org/10.1172/JCl127460.
DEK (a bulky, charged, and hydrophilic protein) has notably been shown to undergo cellular uptake and nuclear localization, mediated by cell surface heparan sulfate proteoglycans (HSPGs) and its nuclear localization signal (NLS), respectively. Intriguingly, internalized DEK maintains its endogenous DNA repair and heterochromatin-stabilizing activities, correcting deficits otherwise present in cells depleted of DEK $(1,12)$.

We implicated endogenous nuclear DEK in regulating hematopoietic stem and progenitor cells (HSCs and HPCs). HPCs in Dek $k^{-1}$ mouse BM are more abundant and proliferative, coincident with decreased long-term competitive and secondary transplant repopulating capacity $(1,13,14)$, suggesting that endogenous DEK modulates hematopoiesis. What role, if any, there is for extracellular DEK in regulating hematopoiesis has not been known. Here we report that extracellular recombinant DEK (rDEK) greatly enhances ex vivo expansion of cytokine-stimulated mouse and human HSCs and modulates HSC and HPC numbers/function in vivo and in vitro, acting through chemokine receptor CXCR2 and utilizing HSPGs as coreceptors. DEK's ability to translocate to the nucleus or bind DNA did not affect hematopoietic regulation in vivo. Consistent with these findings, rDEK-treated mouse BM cells showed increased activation of several factors downstream of CXCR2, including ERK, protein kinase B (AKT), and p38 MAPK. RNA-Seq analysis of rDEK-treated $\mathrm{Dek}^{-/}$lineage-negative ( Lin $\left.^{-}\right) \mathrm{BM}$ cells supported these findings that the major pathways activated by rDEK involved cytokine/chemokine signaling. 


\section{Results}

rDEK enhances ex vivo expansion of mouse BM and human cord blood HSCs. Enhancing ex vivo expansion of HSCs has the potential to improve the efficacy of clinical hematopoietic cell transplantation (HCT), especially for cord blood (CB), since HSC numbers are limited in single CB collections $(15,16)$. We assessed the effects of rDEK on cytokine-stimulated ex vivo expansion of mouse and human HSCs. Culturing mouse Lin ${ }^{-} \mathrm{BM}$ cells in expansion medium containing recombinant mouse ( $\mathrm{rm}$ ) stem cell factor ( $\mathrm{rmSCF}$ ), $\mathrm{rm}$ thrombopoietin (rmTPO), and $\mathrm{rm}$ fms-related tyrosine kinase 3 ligand (rmFlt3L) with rmDEK for 4 days resulted in an approximately 2.8-fold increase in long-term HSC (LT-HSC) (Lin ${ }^{-S c a-1}{ }^{+}$ c-Kit ${ }^{+}$[LSK] CD34-CD150 ${ }^{+} \mathrm{CD} 48^{-} \mathrm{CD}^{-} 1^{-}$) numbers (Figure $1 \mathrm{~A} ; n=$ 6 experiments). Limiting dilution analysis compared frequencies of competitive repopulating units (CRU) in day 0 uncultured cells (input) and progeny of equivalent numbers of cells in the presence of rmDEK or vehicle control after 4 days in culture (Figure 1, B-F). DEK-cultured cells demonstrated significantly greater engraftment in peripheral blood (PB) and BM compared with input and vehicle control cells in primary and secondary transplants (Figure 1, B-D, and Supplemental Figure 1, A-C). Analysis of 4-month $\mathrm{BM}$ in primary transplanted mice revealed a CRU frequency of 1:66,709 in uncultured mouse BM Lin' cells, 1:50,878 in vehicle control cultures, and 1:14,996 in cultures with rmDEK (Figure 1, E and F, and Supplemental Table 1, A and B). Thus, DEK resulted in an approximately 4.5 -fold increase in CRU compared with that in input cells and an approximately 3.4-fold increase compared with that in vehicle-cultured cells (Figure $1 \mathrm{~F}$ ).

DEK was also evaluated for effects on human $\mathrm{CD} 34^{+} \mathrm{CB}$ cells cultured 4 days with recombinant human (rh) SCF (rhSCF), rhTPO, and rhFlt3L with $50 \mathrm{nM}$ rhDEK or vehicle control. DEK induced an approximately 2.5-fold increase in HSC ( $\mathrm{Lin}^{-}$ $\mathrm{CD} 4^{+} \mathrm{CD} 38^{-} \mathrm{CD} 45 \mathrm{RA}^{-} \mathrm{CD} 90^{+} \mathrm{CD} 49 \mathrm{f}^{+}$) expansion (Figure $1 \mathrm{G} ; n$ $=6$ experiments). Human SCID-repopulating cells (SRCs) were assessed on day 0 (input), and progeny of an equivalent number of cells in the presence of DEK or vehicle control assessed after 4 days (Figure 1, H-L). rhDEK-cultured cells showed significantly greater engraftment in $\mathrm{PB}$ and $\mathrm{BM}$ compared with input cells in primary and secondary transplants (Figure $1, \mathrm{H}-\mathrm{J}$, and Supplemental Figure 1, D-F). Analysis of 4-month BM revealed an SRC frequency of 1:5612 in uncultured cells, 1:10,990 in vehicle control cultures, and 1:1327 in cultures containing DEK (Figure 1, K and L, and Supplemental Table 1, C and D). Thus, rhDEK resulted in an approximately 4.2 fold increase in SRC compared with input cells and an approximately 8.3-fold increase compared with vehicle control (Figure 1L). Extracellular DEK thus significantly enhanced cytokine-stimulated ex vivo expansion of functional engrafting mouse and human HSCs.

Exogenous rmDEK alters in vivo hematopoiesis. A role for secreted extracellular DEK in regulating hematopoiesis in vivo has not to our knowledge previously been characterized. Since C57BL/6 BM HSCs and HPCs have endogenous nuclear DEK, we examined the effects of rmDEK on hematopoiesis in $\mathrm{Dek}^{-/}$and WT littermate mice. Ten micrograms of rmDEK or vehicle control was injected s.c. into mice once a day for 2 days, and BM was harvested 48 hours later. rmDEK significantly increased numbers of LSK CD34-Flt3- LT-HSCs in WT ( 46\%) and ${ }^{-} e^{-/-}$mice ( $84 \%$;
Figure $2 \mathrm{~A})$. Increased numbers of LSK CD $34^{+}{ }^{+} \mathrm{Flt}^{-}$short-term HSCs (ST-HSCs) ( $50 \%$ increase; Figure $2 \mathrm{~B})$ and LSK CD $34^{+}{ }^{+} \mathrm{Flt}^{+}$ multipotent progenitors (MPPs; $43 \%$ increase; Figure $2 \mathrm{C}$ ) were observed in $\mathrm{Dek}^{-/}$but not WT treated mice. Thus, $\mathrm{Dek}^{-/-}$mice were more sensitive to exogenous DEK effects than WT mice. In contrast to phenotyped HSCs, absolute numbers of functional CFU-granulocytes, monocytes (CFU-GMs); burst-forming uniterythroid cells (BFU-Es); and CFU-granulocytes, erythrocytes, monocytes, megakaryocytes (CFU-GEMMs) per femur were reduced, respectively, by approximately $73 \%, 77 \%$, and $68 \%$ in $\mathrm{Dek}^{-/-}$mice (Figure 2, D-F). A decrease in cycling HPCs was determined by a high-specific-activity tritiated thymidine $\left({ }^{3} \mathrm{HTdr}\right)$ kill assay (Supplemental Figure 2, A-C). While $D e k^{-/-}$mice had more CFU-GMs ( 2.1-fold increase), BFU-Es ( 2.7 -fold increase), and CFU-GEMMs ( 2.2-fold increase) when compared with WT BM at baseline, they had equivalent numbers when WT and $D e k^{-/-}$mice were treated with DEK.

To control for effects of DEK preparations and to determine whether effects were reversible in vivo, we injected WT C57BL/6 mice s.c. with $10 \mu \mathrm{g}$ heat-denatured rmDEK (drmDEK), rmDEK, or vehicle control once a day for 2 days. BM of C57BL/6 mice treated with rmDEK had reduced numbers of CFU-GMs $(\sim 54 \%$ reduction), BFU-Es ( $46 \%$ reduction), and CFU-GEMMs ( $44 \%$ reduction) at 24 and 48 hours after final injection of DEK, which correlated with decreased cycling of CFU-GMs, BFU-Es, and CFU-GEMMs (Figure 2, G-I, and Supplemental Figure 2, D-F), while at 72 hours a slight reduction in DEK inhibition of HPC numbers and cycling (Supplemental Figure 3, A and B) was noted, with suppressive effects absent by 96 (Supplemental Figure 3, C and D) and 144 hours (Supplemental Figure 3, E and F). Thus, in vivo effects of rmDEK were reversible. Heat-inactivated drmDEK had no significant effect on colony formation (Figure 2, G-I).

To assess effects of in vivo DEK treatment on HSC function, we performed competitive transplantation using donor BM cells collected 24 or 48 hours after vehicle or rmDEK administration to $\mathrm{C} 57 \mathrm{BL} / 6$ mice $\left(\mathrm{CD} 45.1^{-} \mathrm{CD} 45.2^{+}\right)$. Donor CD 45.1 $1^{-} \mathrm{CD} 45.2^{+}$cells in $\mathrm{PB}$ of host $\mathrm{F}_{1}$ mice receiving $\mathrm{BM}$ isolated 24 hours after $\mathrm{rmDEK}$ treatment had decreased engraftment at all time points examined (Figure 2J). However, donor BM cells collected 48 hours following DEK showed only minor decreases in engraftment 2 months after transplantation and no effects at later times (Figure $2 \mathrm{~K}$ ). This suggested that exogenous DEK resulted in time-dependent negative regulatory effects on engrafting HSCs. Thus, increased numbers of phenotypically recognizable HSCs were not reflective of their engrafting capability when rmDEK was given in vivo. Therefore, we examined whether BM collected from DEK-treated mice would show any defect in its ability to home to the host BM following transplantation. There was no significant change in the percentage of LSK CD150 ${ }^{+}$, LSK, and LK cells that were CXCR4 ${ }^{+}$ (Figure 3, A-C). However, CXCR4 ${ }^{+}$LSK CD150 ${ }^{+}, \mathrm{CXCR}^{+}$LSK, and $\mathrm{CXCR}^{+} \mathrm{LK}$ cell populations demonstrated decreased CXCR4 levels when collected 24, but not 48, hours following final injection of rmDEK. This decrease in CXCR4 expression 24 hours after in vivo DEK treatment was associated with decreased homing to host BM (Figure 3, D-F). When examining the BM cells of these same mice, we saw no change in CXCR2 levels (data not shown). Thus, DEK-treated mice manifested increased HSC 

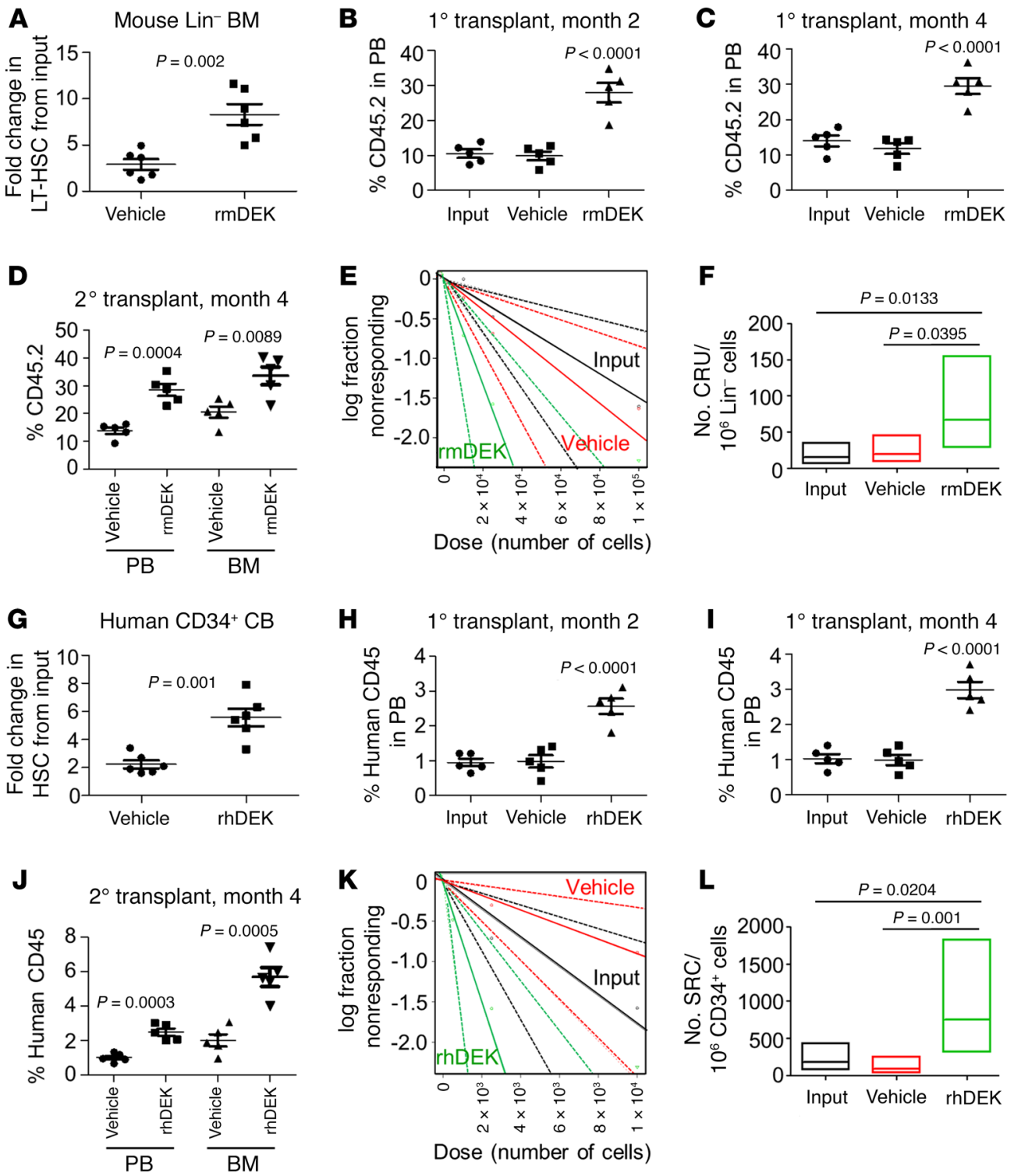

Figure 1. Recombinant DEK enhances ex vivo expansion of mouse Lin- $B M$ and human CD34+ $C B$ HSCs. (A)

Four-day HSC expansion assays using C57BL/6 Lin- BM cells. Data represent mean \pm SEM fold change from input LT-HSC numbers of 6 pools of 2 mice ( $t$ test). (B and C) Donor cells (CD45.2+) from $\mathbf{A}$ and competitor Boy/J BM cells were infused into $\mathrm{F}_{1}$ recipients ( $n=5$ mice/group). $1^{\circ}$, primary; $2^{\circ}$, secondary. Percentages of donor cells in PB were examined after 2 (B) and 4 (C) months. $P$ value compares the indicated group with day 0 input. (D) Secondary BM transplants using mice from $\mathbf{B}$ and $\mathbf{C}$ as donors. Percentages of donor cells were examined at 4 months $(n=5$ mice/group). For B-D, 1-way ANOVA with post hoc Tukey's multiple-comparisons test was performed. (E) Poisson statistical analysis from the limiting dilution analysis. Different doses of donor cells from $\mathbf{A}$ and competitor cells were infused into $F_{1}$ recipients. Symbols represent the percentage of negative mice for each dose of cells. Solid lines indicate the best-fit linear model for each data set. Dotted lines represent $95 \%$ confidence intervals. (F) CRU in $1 \times 10^{6}$ transplanted cells calculated from E. (C) Four-day HSC expansion assays using human CD $34^{+}$CB cells. Data represent mean \pm SEM fold change from input HSC numbers for 6 individual CBs ( $t$ test). (H and I) Donor cells from G were infused into NSG recipients ( $n=5$ mice/group). Percentage of donor human $C D 45^{+}$cells in PB was examined after 2 (H) and 4 (I) months. $P$ value compares indicated group with day 0 input. (J) Secondary BM transplants using mice from $\mathbf{H}$ and $\mathbf{I}$ as donors. Percent donor cells were examined at 4 months. For $\mathbf{H}-\mathbf{J}, \mathbf{1}$-way ANOVA with post hoc Tukey's multiple-comparisons test was performed. (K) Poisson statistical analysis from the limiting dilution analysis utilizing mice from $\mathbf{H}-\mathbf{I}$. (L) Number of SRCs in $1 \times 10^{6}$ transplanted cells was calculated from $\mathbf{K}$.

administered DEK that have not yet been elucidated.

$D E K$ effects are dependent on CXCR2 and Gai protein signaling. DEK has a Glu-Leu-Arg (ELR) motif (5), similar to CXC chemokines such as IL-8 (CXCL8) and macrophage inflammatory protein 2 (MIP2; CXCL2) (17). Like DEK, IL-8 and MIP2 suppress HPC proliferation (18-20). $\mathrm{Dek}^{-/-}$HPCs were more sensitive to $\mathrm{rmDEK}$ inhibition than WT HPCs (Figure 4A), and were more sensitive to rhIL-8 (Figure 4B) and rmMIP2 (Figure 4C). Two single-stranded DNA-targeting aptamers, DTA-64 and DTA-85+ends, that bind tightly to rmDEK, but not a control aptamer library (8), neutralized inhibition by rmDEK but not by rhIL-8, rmMIP2, or CC chemokine MIP1 $\alpha$ (CCL3; Figure 4D), demonstrating specificity of DEK action.

CXCR2 is a negatively acting receptor regulating HPC proliferation and IL-8- and MIP2mediated suppression of hematopoiesis in vitro and in vivo (21, 22). Since DEK contains an ELR motif and, like ELR-containing chemokines, negatively regulates HPC numbers, we hypothesized that CXCR2 is a candidate receptor for extracellular DEK. C57BL/6 BM cells were pretreated in vitro with rat IgG (isotype control) or neutralizing antibodies against CXCR2 or CXCR4 (a non-ELR-binding CXC chemokine receptor that binds CXCL12) prior to being placed in a growth factor-dependent HPC colony assay containing rmDEK, rhIL-8, rmMIP2, rmMIP1 $\alpha$, or vehicle control (Figure 4E). Blocking CXCR2 neutralized inhibition by $\mathrm{rmDEK}$, rhIL-8, and rmMIP2, but not that by rmMIP1 $\alpha$ (a nonELR motif-containing chemokine thats does not bind CXCR2). Thus, rmDEK, like IL-8 and numbers, but with overall BM engrafting deficiencies associated, at least in part, with decreased CXCR4 expression and homing efficiency. The differences in DEK's effect ex vivo and in vivo on engrafting HSCs was likely due to other in vivo effects in mice
MIP2, requires CXCR2 for myelosuppression. Blocking CXCR4 did not affect actions of rmDEK or the other chemokines. To determine whether rmDEK function requires CXCR2 in vivo, we injected $10 \mu \mathrm{g}$ rmDEK or vehicle control s.c. once a day for 2 days into 

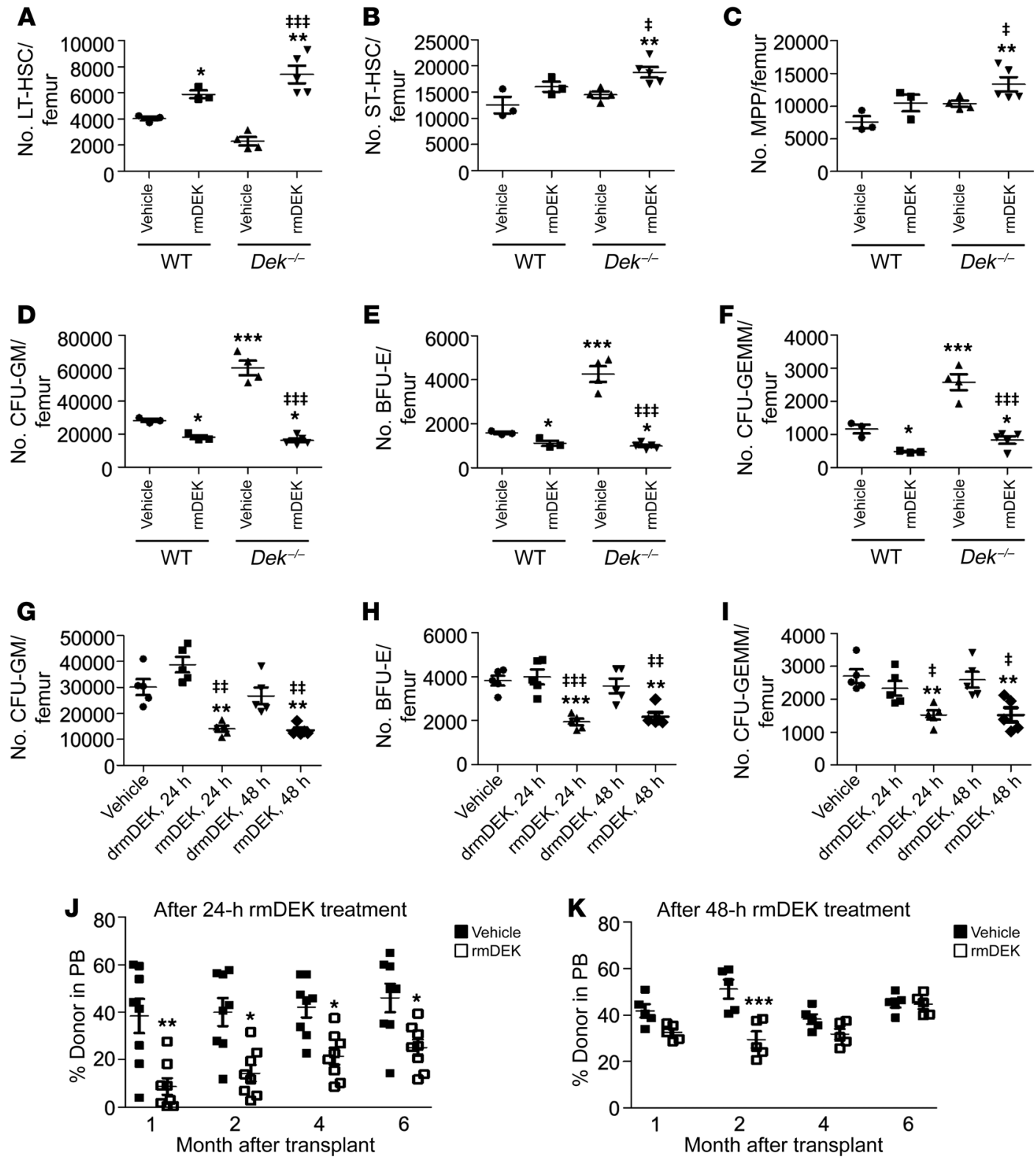

Figure 2. Administration of rmDEK regulates hematopoiesis in vivo. (A-F) $D e k^{-1-}$ or littermate WT control mice were injected s.c. with $10 \mu \mathrm{g}$ dialyzed rmDEK or vehicle once a day for 2 days ( $n=3-5$ mice/group). BM was harvested 48 hours after final injection. Immunophenotyping of LT-HSCs (A), ST-HSCs (B), and MPPs (C) was performed using flow cytometry. Data are mean \pm SEM. (D-F) HPC numbers per femur were determined by CFU assay. Data are mean \pm SEM of individually assessed mice per group plated in triplicate. ${ }^{*} P<0.05,{ }^{* *} P<0.01,{ }^{* * *} P<0.001$ compared with WT vehicle control; ${ }^{\ddagger} P<0.05$, ${ }^{\prime \neq \ddagger} P<0.001$ compared with vehicle-treated Dek $k^{-1-}$ mice. (G-I) C57BL/6 mice were given $10 \mu \mathrm{g} \mathrm{rmDEK,} \mathrm{drmDEK,} \mathrm{or} \mathrm{vehicle} \mathrm{control} \mathrm{as} \mathrm{in} \mathrm{A-F,} \mathrm{then} \mathrm{BM} \mathrm{was} \mathrm{harvested} 24$ and 48 hours after final injection. HPC numbers were determined. Data are mean \pm SEM of 5 individually assessed mice per group plated in triplicate. ${ }^{* *} P<0.01$, ${ }^{*}{ }^{*} P<0.001$ compared with WT vehicle control; ${ }^{\ddagger} P<0.05$, ${ }^{\ddagger} P<0.01,{ }^{\ddagger \neq \ddagger} P<0.001$ compared with drmDEK group. (J) Engrafting efficiency of donor $C 57 \mathrm{BL} / 6$ BM cells collected 24 hours following final injection of vehicle or rmDEK (once a day for 2 days) in PB. Data are mean \pm SEM of 8 host mice. (K) Engrafting efficiency of donor C57BL/6 BM cells collected 48 hours following final injection of vehicle or rmDEK (once a day for 2 days) in PB. ${ }^{*} P<0.05$, ${ }^{* *} P<0.01$, ${ }^{* * *} P$ $<0.001$ compared with vehicle-treated group at the same time point. For A-K, 1-way ANOVA with post hoc Tukey's multiple-comparisons test was used.

Cxcr2 ${ }^{-/-}$or littermate WT control mice. BM was harvested 48 hours later. As shown in Figure 2A, WT mice receiving rmDEK had an approximately $42 \%$ increase in phenotyped LT-HSCs compared with vehicle-treated WT mice (Supplemental Figure 4A), with no changes in ST-HSCs (Supplemental Figure 4B) and MPPs (Supplemental Figure 4C). However, rmDEK had no effect on LT-HSC, ST-HSC, or MPP numbers in $\mathrm{Cxcr} 2^{-/-}$mice, demonstrating that CXCR2 is required for the DEK-mediated enhancement of phe- notyped LT-HSC numbers in vivo. As reported (22), cycling HPC numbers in $\mathrm{Cxcr}^{-/-}$mice were significantly increased compared with those in WT mice (Figure 4, F-H, and Supplemental Figure $4, \mathrm{D}-\mathrm{F}$ ) but were unaffected by rmDEK treatment, confirming DEK-mediated effects through CXCR2.

Dipeptidyl peptidase 4 (DPP4), a serine protease that cleaves select penultimate amino acids of proteins, truncates and downmodulates functional activities of select cytokines and chemo- 

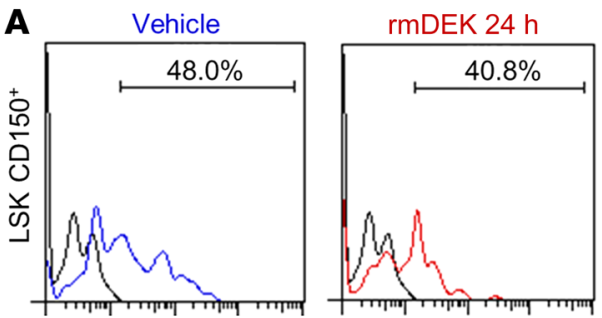

APC-CXCR4
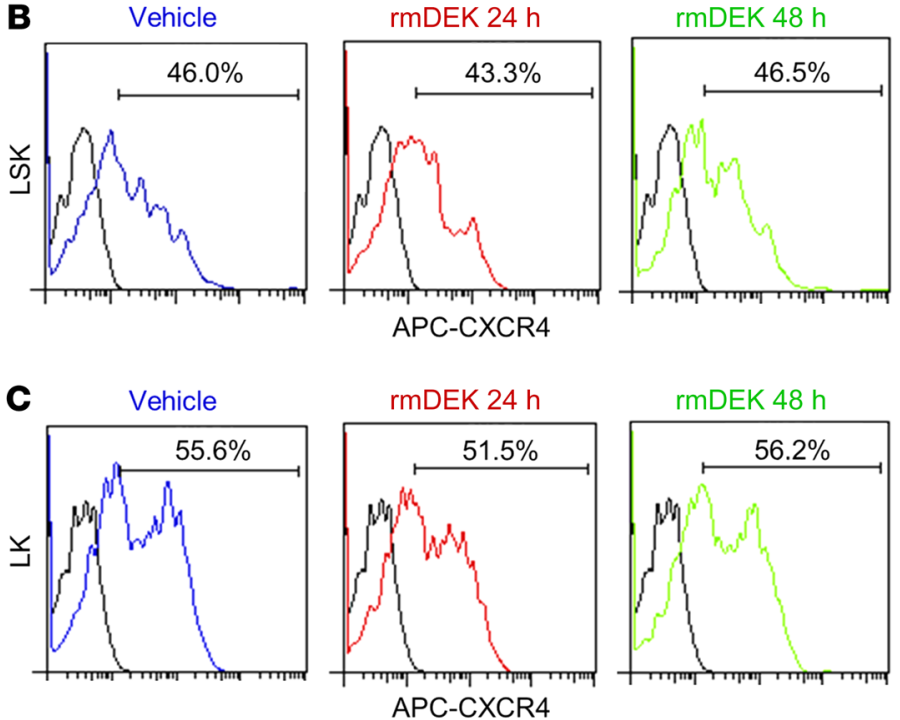
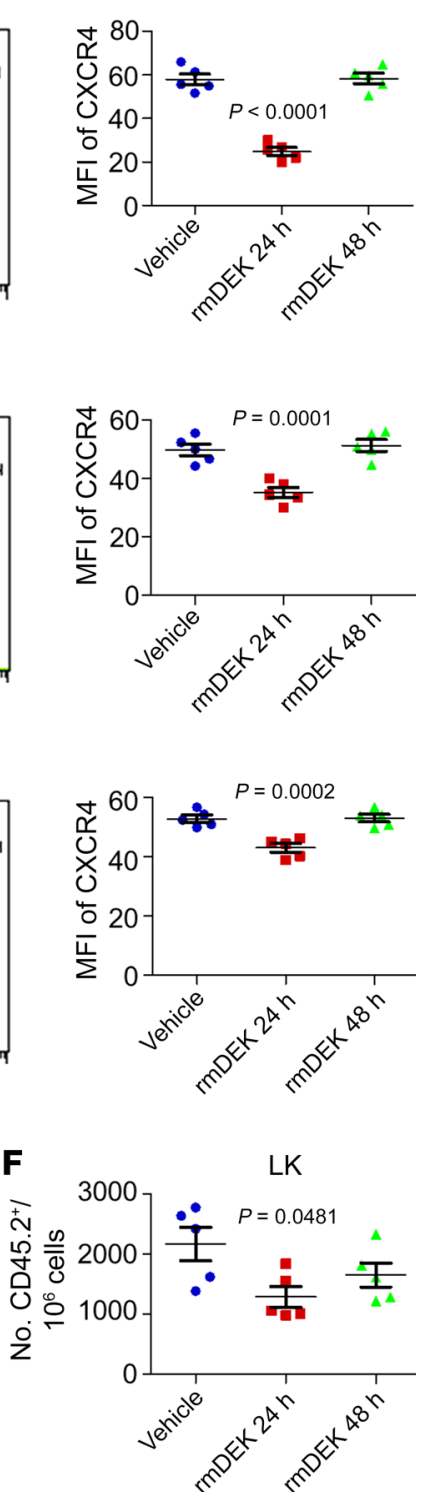

Figure 3. In vivo rmDEK treatment reversibly decreases CXCR4 expression and homing capability of BM cells. C57BL/6 mice (CD45.1-CD45.2 $)$ were injected with $10 \mu$ g dialyzed WT rmDEK or vehicle control s.c. once a day for 2 days. BM was then collected 24 or 48 hours after final injection. (A-C) CXCR4 expression levels were examined 24 and 48 hours following the final DEK injection by flow cytometry in the CD45.1-CD45.2 $2^{+}$LSK CD150+ (A), LSK (B), and LK (C) cell populations. (D-F) Twelve million cells from the mice examined in $\mathbf{A}-\mathbf{C}$ were injected i.v. into lethally irradiated (950 cGy, 24 hours prior) B6×Boy/J F mice (CD45.1 $1^{+}$D 45.2 $\left.2^{+}\right)$hosts. Eighteen hours following injection, BM from host mice was collected and analyzed by flow cytometry for the presence of CD45.1-CD45.2+ LSK CD150+ (D), LSK $(\mathbf{E})$, and LK (F) cells. In $\mathbf{A}-\mathbf{F}$, data are mean \pm SEM of 5 mice per group; $P$ value is compared with vehicle control-treated mice using 1-way ANOVA with post hoc Tukey's multiple-comparisons test.
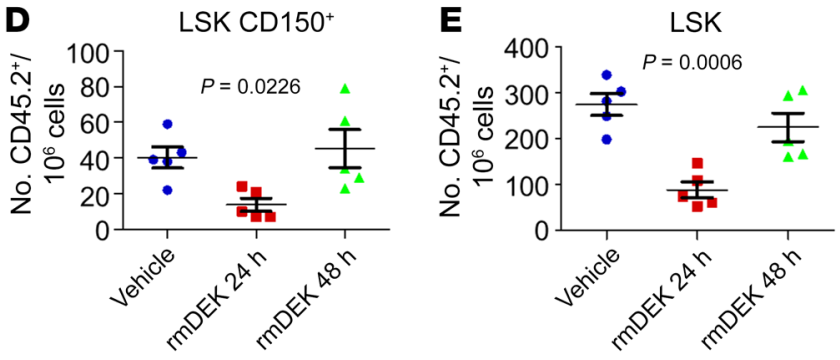

kines. DPP4-truncated molecules downmodulate the effectiveness of their own full-length forms, effects mediated at the receptor level (23-25). DEK and IL-8 have DPP4 truncation sites $(14,25)$. Upon DPP4 truncation, DPP4 inactivates IL-8 (21) and rmDEK in vitro (Figure 4I). Interestingly, DPP4-truncated DEK and IL-8 counteracted the suppressive activities of their respective full-length protein, as well as the other full-length protein, as effectively as anti-CXCR2. This strongly suggests that DEK and IL-8 share the same receptor, CXCR2.

As selected myelosuppressive chemokines do not require Gai protein signaling to mediate inhibitory effects (26), we examined whether rmDEK requires Gai protein signaling to reduce HPC numbers. C57BL/6 BM cells were pretreated with pertussis toxin (PT), and suppression by rmDEK, rhIL-8, rmMIP2, rmMIP1 $\alpha$, and rm6Ckine was examined via HPC colony assay (Figure 4J). Pretreatment with PT did not block rhIL-8, rmMIP2, rmMIP1 $\alpha$, or rm6Ckine inhibition of HPC colony formation, but did interfere with DEK-mediated inhibition of HPC colony formation, suggesting that DEK, unlike other myelosuppressive chemokines (includ- ing those working through CXCR2), requires Gai protein signaling to mediate myelosuppression. This suggests that while DEK, IL-8, and MIP2 work through the same receptor, CXCR2, the initiation of intracellular signaling by DEK may be different.

DEK requires HSPGs to regulate HSC and HPC numbers. Charged hydrophilic DEK binds to negatively charged HSPGs on the surface of HeLa cells (12). To determine whether DEKmediated myelosuppression requires DEK binding to HSPGs, we pretreated C57BL/6 BM cells with Xyl-PheNO 2 ( $p$-nitrophenyl-o- $\beta$-D-xylopyranoside; disrupts proteoglycan biosynthesis) or heparinase III (cleaves HSPGs from the cell surface) prior to HPC colony assay in the presence of rmDEK or vehicle control (Figure 5A). DEK-mediated myelosuppression was blocked with Xyl-PheNO $\mathrm{N}_{2}$ or heparinase III, suggesting that DEK requires HSPGs to mediate myelosuppression. To determine whether HSPGs or CXCR2 are necessary for DEK-mediated enhanced ex vivo expansion of mouse BM LT-HSCs, we pretreated mouse Lin $^{-} \mathrm{BM}$ cells with heparin (blocks binding of charged molecules to HSPGs), neutralizing CXCR2 antibody, isotype control, or 
A 250

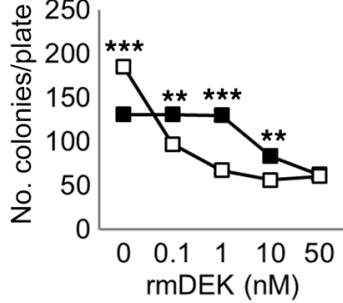

B $\nsubseteq 250$

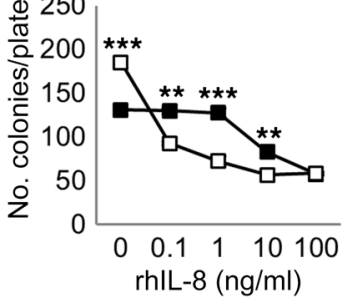

C

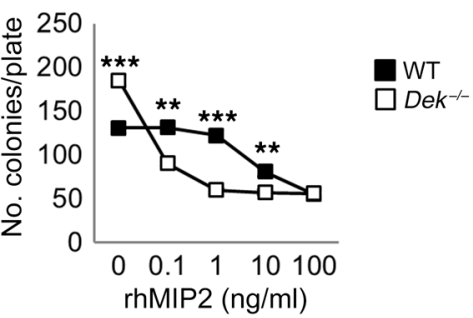

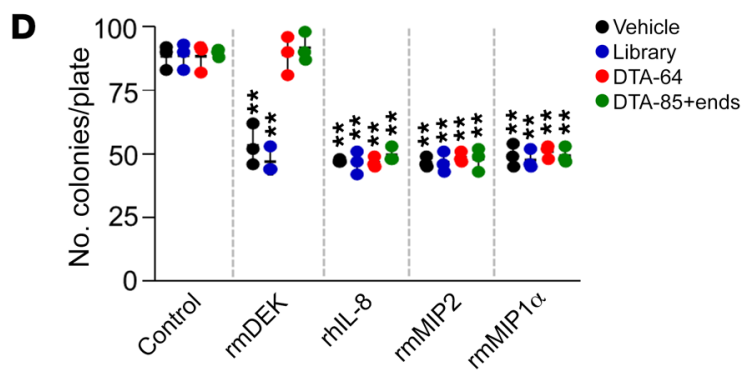
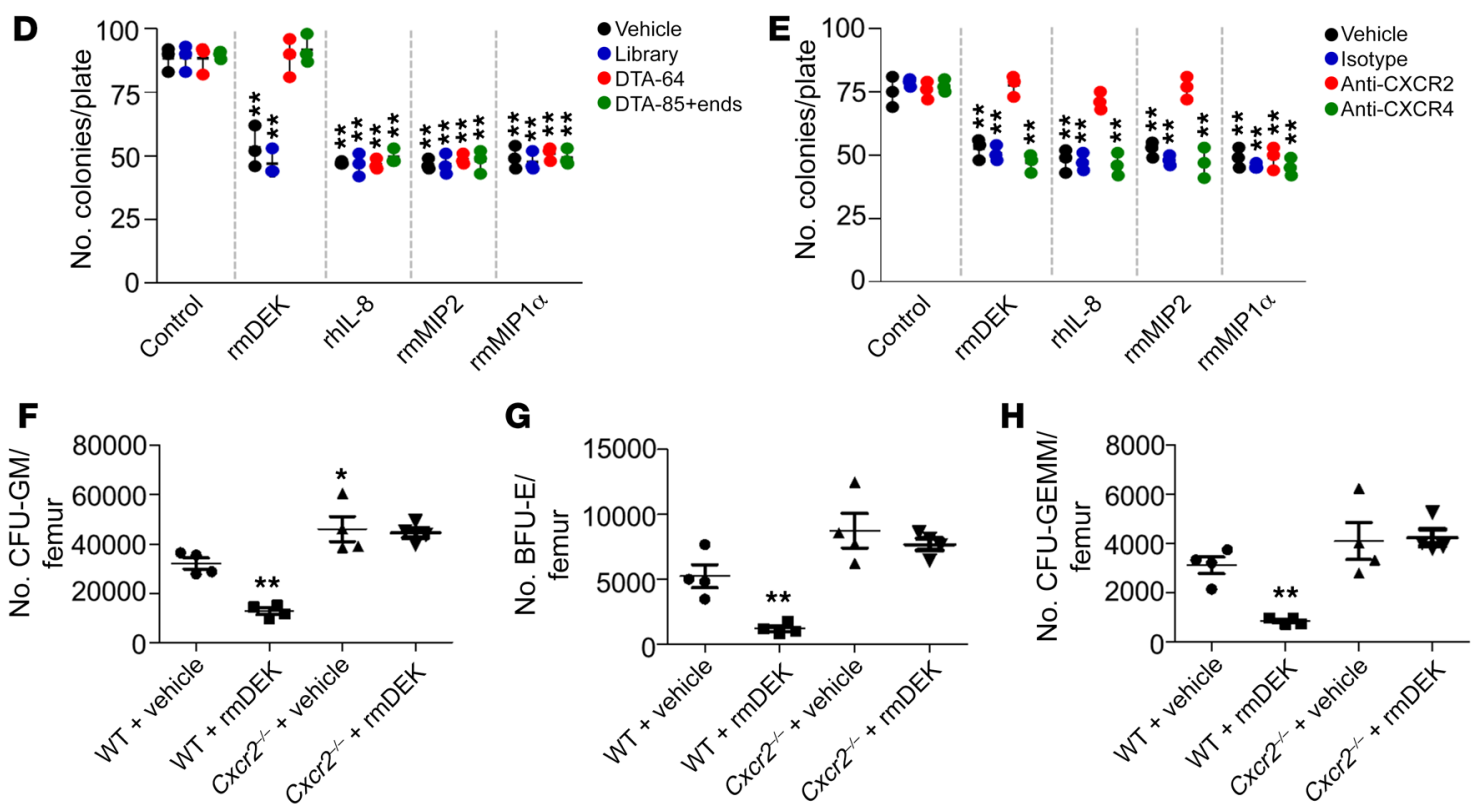

I
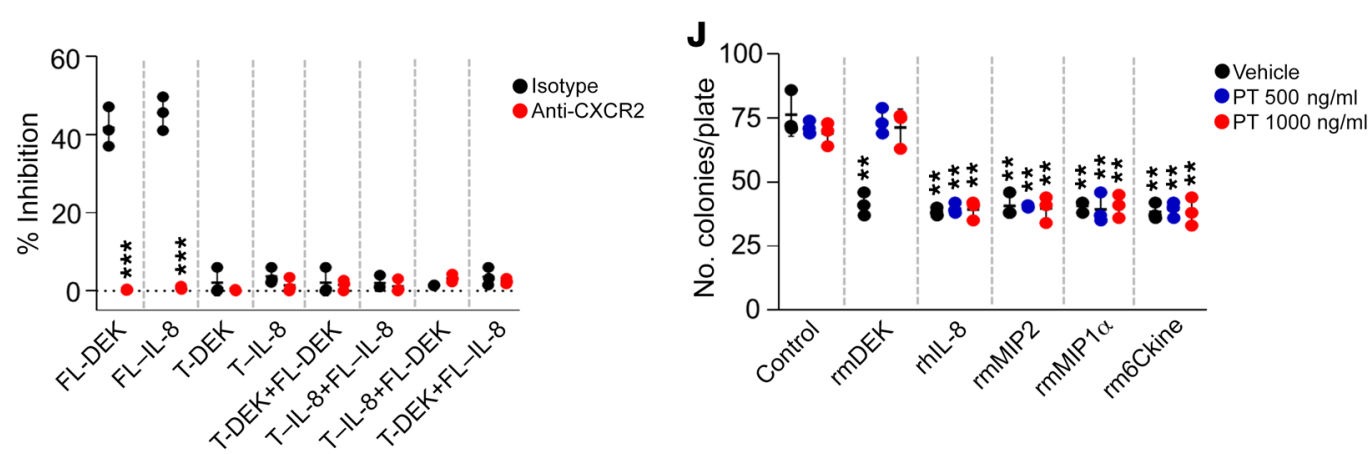

Figure 4. Inhibition of HPC numbers by DEK is CXCR2 and Gai dependent. (A-C) HPC CFU assays with rmDEK (A), rhIL-8 (B), or rmMIP2 (C) using either Dek $k^{-1}$ or WT BM cells. ${ }^{* *} P<0.01,{ }^{* * *} P<0.001$ comparing doses between WT and Dek ${ }^{-1-}$ BM cells. (D) HPC CFU assay examining the effect of pretreating rmDEK, rhIL-8, rmMIP2, or rmMIP1 $\alpha$ with DEK-targeting aptamers (DTA-64 and DTA-85+ends) or control vehicle and DTA library. (E) HPC CFU assay examining whether pretreatment of WT BM cells with anti-CXCR2 blocking antibody inhibits rmDEK, rhIL-8, rmMIP2, or rmMIP1 $\alpha$ effects. Vehicle, isotype antibody, and anti-CXCR4 blocking antibody were used as controls. ${ }^{* *} P<0.01$ compared with control. In $\mathbf{A}-\mathbf{E}$, data are mean \pm SD of triplicate plates. (F-H) $C x \mathrm{Cr}^{-1-}$ or WT mice were injected s.c. with $10 \mu \mathrm{g}$ dialyzed rmDEK or vehicle once a day for 2 days. BM was harvested 48 hours after final injection. HPC number was determined by CFU assay. Data are mean \pm SEM of 4 mice per group plated in triplicate. ${ }^{*} P<0.05,{ }^{* *} P<0.01$ compared with vehicle-treated WT mice. (I) HPC CFU assay examining whether full-length (FL) or DPP4-truncated (T) rmDEK or IL-8 alone or mixed at a 1:1 ratio affects C57BL/6 BM colony formation when pretreated with anti-CXCR2 blocking antibody or isotype control. Percent inhibition was calculated based on counts from plates with control media added. (J) HPC CFU colony assay using C57BL/6 BM cells that were pretreated with PT (Gai inhibitor) or vehicle control, then cultured with rmDEK, rhIL-8, rmMIP2, rmMIP1 $\alpha$, or rm6Ckine. In I and J, data are mean \pm SD of triplicate plates. ${ }^{* *} P<0.01,{ }^{* * *} P<0.001$ compared with control. For A-J, 1-way ANOVA with post hoc Tukey's multiple-comparisons test was used.

vehicle control prior to ex vivo expansion. Pretreating mouse LinBM cells with heparin or neutralizing CXCR2 antibody blocked DEK-mediated enhancement of LT-HSC numbers (Figure 5B), suggesting that DEK-mediated enhancement of LT-HSCs ex vivo is CXCR2 and HSPG dependent.
DEK-mediated trimethylation of H3K9 in LSK cells requires HSPGs. Extracellular DEK is internalized and remodels chromatin, as indicated by increased trimethylation of H3K9 in DEK-knockdown HeLa cells in vitro $(2,12)$. Previous studies on nonhematopoietic cells suggested that DEK mediates its function, at least 
in part, through regulation of heterochromatin integrity (12). We explored whether extracellular DEK similarly regulated LSK cell function. $\mathrm{Dek}^{-/-}$and littermate WT Lin ${ }^{-} \mathrm{BM}$ cells were cultured for 16 hours with rmDEK or vehicle control, then LSK cells were assessed by imaging flow cytometry for changes in expression of the nuclear heterochromatin marker H3K9me3 (Figure 5C). There was no change in H3K9me3 expression in the nucleus of WT LSK cells following rmDEK treatment, as determined by comparing the similarity scores (calculated using IDEAS software) of cells stained with H3K9me3 and the nuclear dye DRAQ5 (data not shown), similar to previous findings that only DEK-knockdown HeLa cells demonstrated changes in $\mathrm{H} 3 \mathrm{~K} 9$ me3 following incubation with rhDEK (12). However, vehicle-treated $D e k^{-/-}$LSK cells had approximately $52 \%$ less H3K9me 3 in the nucleus compared with WT LSK cells (Figure 5D), and pretreatment of $\mathrm{Dek}^{-/-}$LSK cells with rmDEK significantly increased H3K9me3 levels.

DEK must bind to HSPGs to become internalized through an active process in DEK-knockdown HeLa cells (12). To determine whether DEK required HSPGs to be internalized, we pretreated $\mathrm{Dek}^{-/-} \mathrm{Lin}^{-} \mathrm{BM}$ cells with heparin (Figure 5D), which inhibited DEK-mediated enhancement in H3K9me3 levels in the nucleus of $D e k^{-/}$LSK cells. Pretreating $D^{-1 /-} \mathrm{Lin}^{-} \mathrm{BM}$ cells with Pitstop 2, an endocytosis inhibitor (27), or PT also inhibited DEK-mediated enhancement in H3K9me3 levels in the nucleus of $\mathrm{Dek}^{-/}$LSK cells. Thus, DEK-mediated changes in H3K9me3 in the nucleus of LSK cells requires an endocytosis event involving Gai protein signaling. Interestingly, only $\mathrm{Dek}^{-/-}$LSK cells that were actively cycling showed major changes in nuclear H3K9me3. Pretreating $\mathrm{Dek}^{-/-} \mathrm{Lin}^{-}$ $\mathrm{BM}$ cells with hydroxyurea prior to culture to kill actively cycling cells and then adding rmDEK resulted in lower nuclear H3K9me3 levels than treatment with vehicle control alone (Figure 5D).

Inactivation of rmDEK with the aptamers DTA-64 or DTA-85+ends, but not a control aptamer library, blocked DEKmediated enhancement of trimethylation of H3K9 (Figure 5E). However, blocking CXCR2 prior to incubation with DEK had no effect on DEK-mediated enhancement of H3K9me3 expression in the nucleus of $\mathrm{Dek}^{-/-}$LSK cells (Figure 5E). Although CXCR2 is required to mediate DEK's effect on hematopoiesis in vivo and in vitro, it is apparently not required for DEK-mediated heterochromatin remodeling in $D e k^{-/-}$LSK cells. This suggested that the heterochromatin remodeling actions of DEK may not be involved in the cytokine-like effects of DEK in regulating hematopoiesis.

Extracellular DEK does not require translocation to the nucleus or binding to DNA to regulate hematopoiesis. To determine whether DEK's nuclear functions are required to mediate rmDEKmediated regulation of hematopoiesis, we utilized 2 mutant forms of rmDEK: DEK that lacks its nuclear translocation signal (rhNLS-DEK) and DEK lacking DNA-binding ability via its major and central DNA-binding motif (SAP-box; rhDBM-DEK). As WT rmDEK is His-tagged and made in baculovirus, but rhNLS-DEK and rhDBM-DEK are GST-tagged and made in E. coli, we included GST-tagged WT rhDEK as another control. As shown in Figure 5, C-E, WT rDEK treatment resulted in increased H3K9me3 expression in the nucleus of $\mathrm{Dek}^{-/-}$LSK cells, and unsurprisingly, rhDBMDEK and rhNLS-DEK did not alter H3K9me3 expression (Figure $6 \mathrm{~A}$ ), suggesting that extracellular DEK must be able to locate to the nucleus and bind to DNA to mediate changes in $\mathrm{H} 3 \mathrm{~K} 9 \mathrm{me} 3$ expression. We examined whether rhDBM-DEK and rhNLS-DEK manifest DEK's suppressive effects on C57BL/6 BM HPC colony formation (Figure 6B). As shown in Figure 4A, WT rDEK dosedependently inhibited HPC colony formation, and rhDBM-DEK and rhNLS-DEK were as efficient as WT rmDEK, suggesting that extracellular DEK's ability to locate to the nucleus and bind to DNA is not required for in vitro inhibition of HPC numbers. The nuclear function of DEK was also not required for DEK-mediated enhancement of mouse LT-HSC numbers following ex vivo expansion (Figure 6C). Both WT rDEKs significantly enhanced mouse LT-HSC numbers after 4 days in culture. rhDBM-DEK ( 2.1-fold increase) and rhNLS-DEK ( 2.6-fold increase) also significantly enhanced LT-HSC expansion, although not nearly as effectively as their GST WT rhDEK counterpart ( $41 \%$ and $\sim 34 \%$ reduction, respectively, from WT DEK).

To determine whether mutant forms of rDEK had effects on regulating hematopoiesis in vivo, we injected C57BL/6 mice s.c. once a day for 2 days with $10 \mu \mathrm{g}$ WT rDEK, rhDBM-DEK, rhNLSDEK, or vehicle control, and analyzed BM 48 hours later. WT rDEK, rhDBM-DEK, and rhNLS-DEK significantly increased LT-HSC numbers to an equivalent extent (Figure 6D), but had no effect on ST-HSC (Figure 6E) or MPP numbers (Figure 6F). WT rDEK, rhDBM-DEK, and rhNLS-DEK were equivalent in suppression of HPC numbers (Figure 6, G-I) when given in vivo to the same WT mice. WT and mutant rDEKs also demonstrated equivalent reductions in percent cycling HPCs (Figure 6, J-L). This suggests that exogenously added DEK does not need to locate to the nucleus, bind DNA, or affect global heterochromatin structure to regulate hematopoiesis in vivo.

DEK stimulates ERK, AKT, and p38 MAPK phosphorylation in LSK CD15O+ and myeloid-enriched progenitor cells. As extracellular DEK's ability to regulate HSC and HPC numbers does not appear to require DEK's nuclear functional activity (but does require CXCR2), we assessed whether DEK activates 4 major proteins downstream of CXCR2: ERK1/2, AKT, p38 MAPK, and NF-кB (28). Lin- BM cells from WT C57BL/6 mice were stimulated with rhIL-8, rmDEK, or vehicle control for 15, 30, and 60 minutes and 16 hours, and LSK CD150 ${ }^{+}$cells and myeloid-enriched progenitor ( Lin $^{-} \mathrm{Sca}-1^{-} \mathrm{c}-\mathrm{Kit}^{+}$or LK) cells were analyzed by flow cytometry for levels of ERK1/2 pT202/pY204 (Figure 7A), AKT pS473 (Figure 7B), or p38 MAPK pT180/pY182 (Figure 7C and Supplemental Figure 5). Like rhIL-8, rmDEK enhanced ERK1/2 and AKT phosphorylation over baseline in these cells. rmDEK achieved peak activation of p38 MAPK pT180/pY182 levels in the LSK CD150+ population after 1 hour of stimulation compared with 15 minutes for rhIL-8. However, p38 MAPK phosphorylation reached its peak in LK cells at 15 minutes following either rhIL-8 or rmDEK treatment, with no observable phosphorylation of ERK1/2, AKT, or p38 MAPK after 16 hours of rhIL-8 or rmDEK stimulation.

To determine whether NF- $\kappa \mathrm{B}$ was being activated following rmDEK treatment, we quantified the degree of NF- $\kappa \mathrm{B}$ p 65 translocation by calculating the similarity of NF- $\mathrm{KB}$ p 65 and nuclear dye DRAQ5 images analyzed by ImageStream flow cytometry. Cells with low similarity scores exhibited little to no correlation between images (corresponding with predominant cytoplasmic distribution of NF-kB p65; s.s. $<1$ ), whereas cells with high similarity scores exhibited positive correlation between the images (corresponding 


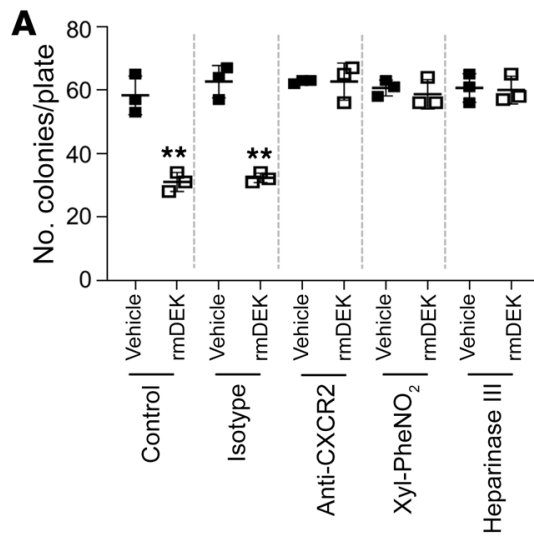

C

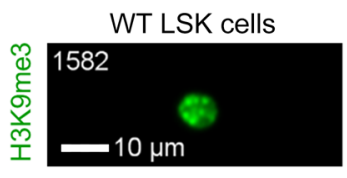

B

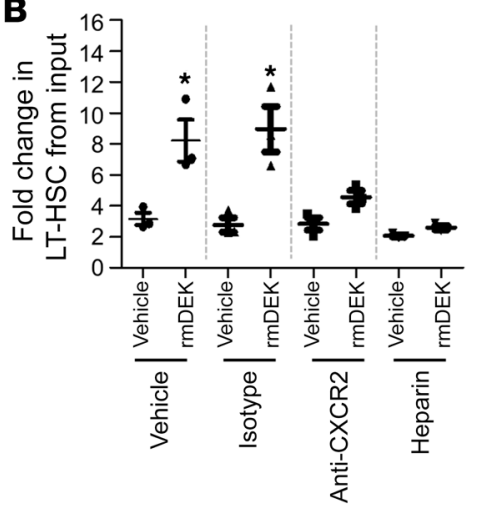

Dek'- LSK cells

$$
+\mathrm{rmDEK}
$$

D

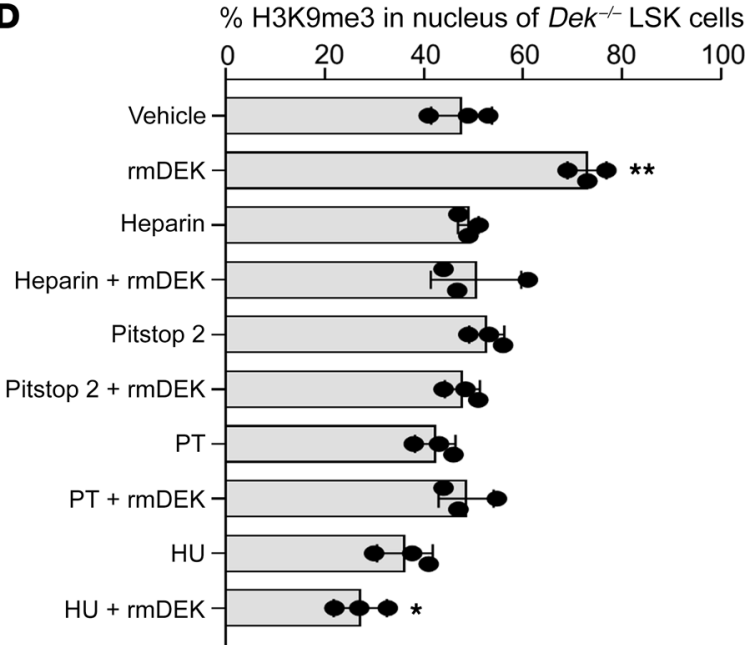

$\mathbf{E}$

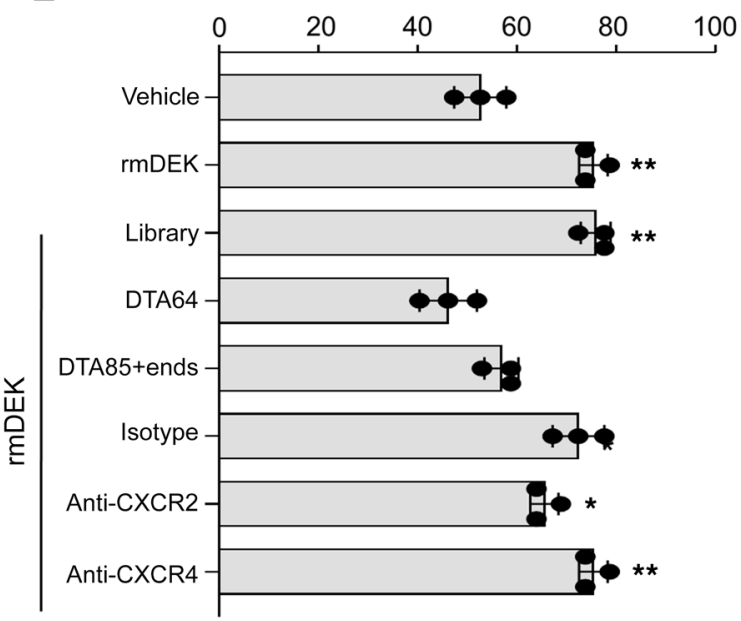

Figure 5. Extracellular DEK's ability to regulate hematopoiesis requires HSPGs. (A) C57BL/6 BM cells were pretreated with $\mathrm{Xyl}^{-} \mathrm{PheNO}_{2}$ or heparinase III to neutralize HSPGs and then utilized in a CFU assay. Anti-CXCR2 antibody served as a positive control, while vehicle or isotype control served as a negative control. Data are mean \pm SD of triplicate plates. ${ }^{* *} P<0.01$ compared with vehicle control. (B) HSC expansion assays using C57BL/6 Lin- BM cells pretreated with anti-CXCR2 blocking antibody or heparin (blocks HSPGs). Isotype antibody or vehicle control served as negative control. Number of LT-HSCs in day 0 input and number of LT-HSCs 4 days after culture in expansion media with either vehicle control or rmDEK were determined. Three pools of 2 mice were utilized. Data are mean \pm SEM. ${ }^{*} P<0.05$ compared with vehicle control. (C) Representative images from Amnis ImageStream analysis of H3K9me3 in the nucleus of WT and vehicle- or rmDEK-treated Dek ${ }^{-1-}$ LSK cells. Images were taken at $\times 40$. Numbers in the upper left are object image numbers. Scale bars: $10 \mu \mathrm{m}$. (D) ImageStream analysis of H3K9me3 in the nucleus of Dek ${ }^{-1-}$ LSK cells pretreated with HSPG inhibitor (heparin), endocytosis inhibitor (Pitstop 2), the Gai protein signaling inhibitor PT, or an agent that kills cycling cells (hydroxyurea [HU]), then incubated for 16 hours with either vehicle or rmDEK. (E) H3K9me3 expression in the nucleus of Dek ${ }^{-1-}$ LSK cells pretreated with anti-CXCR2 blocking antibody, anti-CXCR4 antibody, isotype antibody, or vehicle, then incubated for 16 hours with either vehicle or rmDEK pretreated with vehicle, aptamer library, or DEK-targeted aptamers DTA-64 and DTA-85+ends. For D and E, IDEAs software was used for analysis. Data are mean $\pm \mathrm{SD}$ of triplicate tubes. ${ }^{*} P<0.05,{ }^{* *} P<0.01$ compared with percent $\mathrm{H} 3 \mathrm{~K} 9 \mathrm{me} 3$ levels in the nucleus of the vehicle group. For A-E, 1-way ANOVA with post hoc Tukey's multiple-comparisons test was used.

with predominant nuclear distribution of NF-kB p65; s.s. >1). LSK and LK cells from rhIL-8- (at 15 and 30 minutes) or rmDEK-treated (at 30 minutes) Lin ${ }^{-}$C57BL/6 BM cells demonstrated moderate NF- $\kappa$ B p65 nuclear translocation (s.s. $>1$; Supplemental Figure 6).

Neutralizing CXCR2 on C57BL/6 Lin- BM cells using antiCXCR2 antibody blocked rhIL-8 and rmDEK enhancement of ERK1/2 and AKT phosphorylation in LSK $\mathrm{CD} 15 \mathrm{O}^{+}$cells, whereas
rmSCF and rhSDF1 $\alpha$ were still able to stimulate, as they did not bind to CXCR2 (Supplemental Figure 7, A-F). Thus, DEK signaling through CXCR2 leads to activation of ERK1/2 and AKT in LSK CD150 ${ }^{+}$BM cells. Blocking HSPGs by pretreating the BM cells with heparin interfered with cytokine-stimulated phosphorylation of ERK1/2 and AKT (Supplemental Figure 7, A-F) suggesting that HSPGs play a vital role in signaling of many cytokines, not 

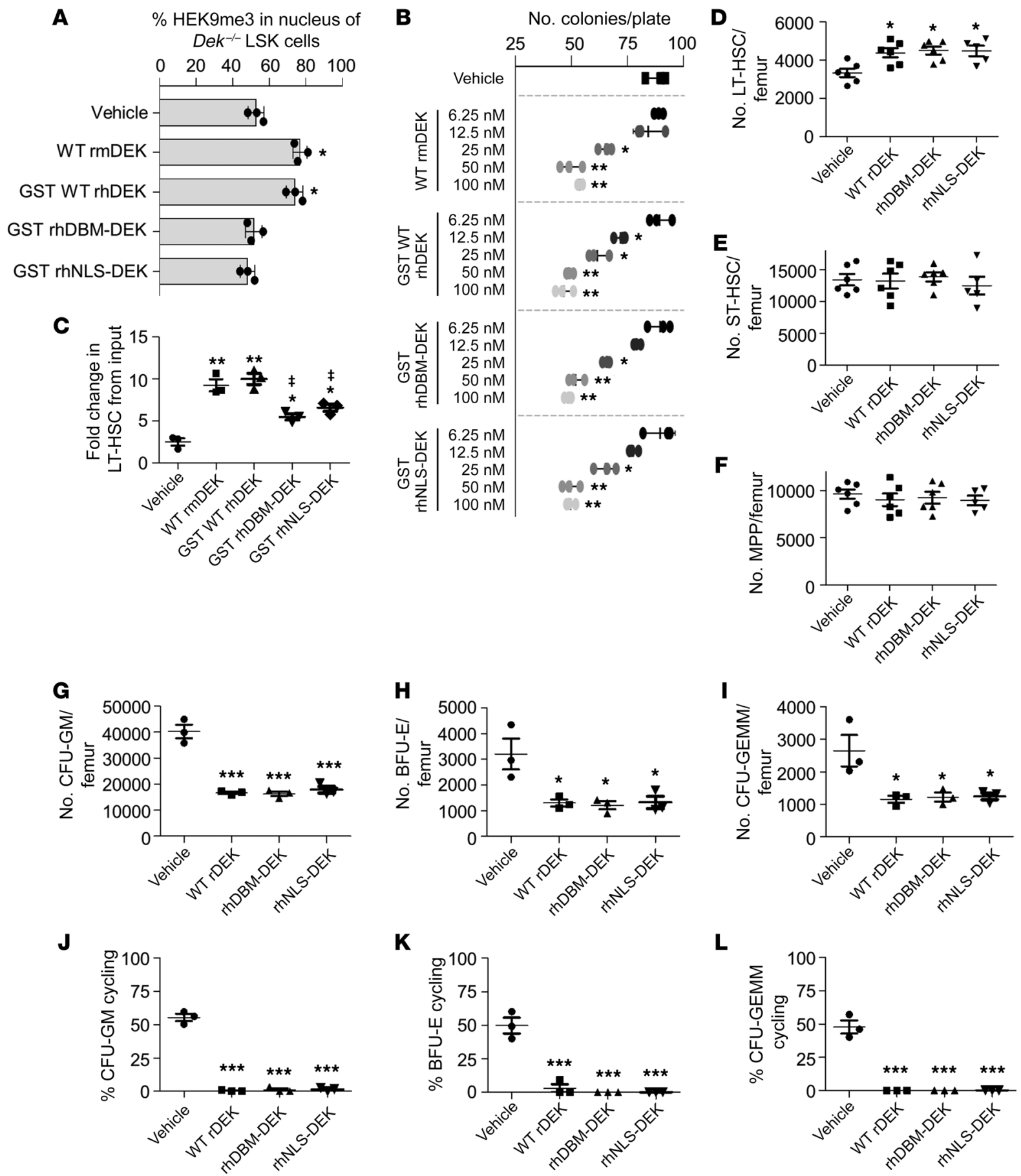

Figure 6. Extracellular DEK does not require its nuclear function to regulate hematopoiesis. (A) Analysis of H3K9me3 levels in the nucleus of Dek ${ }^{-1-}$ LSK cells incubated with vehicle, WT rmDEK, GST-tagged WT rhDEK (CST WT rhDEK), GST-tagged mutant DNA-binding motif rhDEK (CST rhDBM-DEK), or GST-tagged mutant NLS rhDEK (CST rhNLS-DEK). Data are mean \pm SD of triplicate tubes. ${ }^{*} P<0.05$ compared with percent $\mathrm{H3K} 9$ me3 levels in the nucleus of the vehicle group. (B) HPC colony assay examining the effect of different doses of WT or mutant DEK. Data are mean \pm SD of triplicate plates. ${ }^{*} P<$ $0.05,{ }^{* *} P<0.01$ compared with vehicle control. (C) Number of LT-HSCs in day 0 input of C57BL/6 Lin- BM cells and number of LT-HSCs 4 days after culture in expansion media with vehicle or WT or mutant DEK was determined. Data are \pm SEM of fold change from input LT-HSC numbers of 3 pools of 2 mice. ${ }^{*} P<0.05,{ }^{* *} P<0.01$ compared with vehicle control; ${ }^{\ddagger} P<0.05$ compared with WT rmDEK or CST WT rhDEK. (D-F) C57BL/6 mice were injected s.c. with vehicle or $10 \mu \mathrm{g}$ dialyzed WT or mutant DEK once a day for 2 days. BM was harvested 48 hours after final injection, and immunophenotyping of LT-HSCs (D), ST-HSCs (E), and MPPs (F) was performed. Data are mean \pm SEM of 6 mice per group. ${ }^{*} P<0.05$ compared with vehicle-treated mice. (G-I) HPC number from the mice used in $\mathbf{D}-\mathbf{F}$ was determined by CFU assay. The percentage of HPCs at the time of isolation in cycle was determined by ${ }^{3} \mathrm{HTdr}$ assays $(\mathrm{J}-\mathbf{L})$. Data are mean \pm SEM of 6 mice per group plated in triplicate. ${ }^{* * *} P<0.001$ compared with vehicle-treated WT mice. For A-L, 1-way ANOVA with post hoc Tukey's multiple-comparisons test was used. 

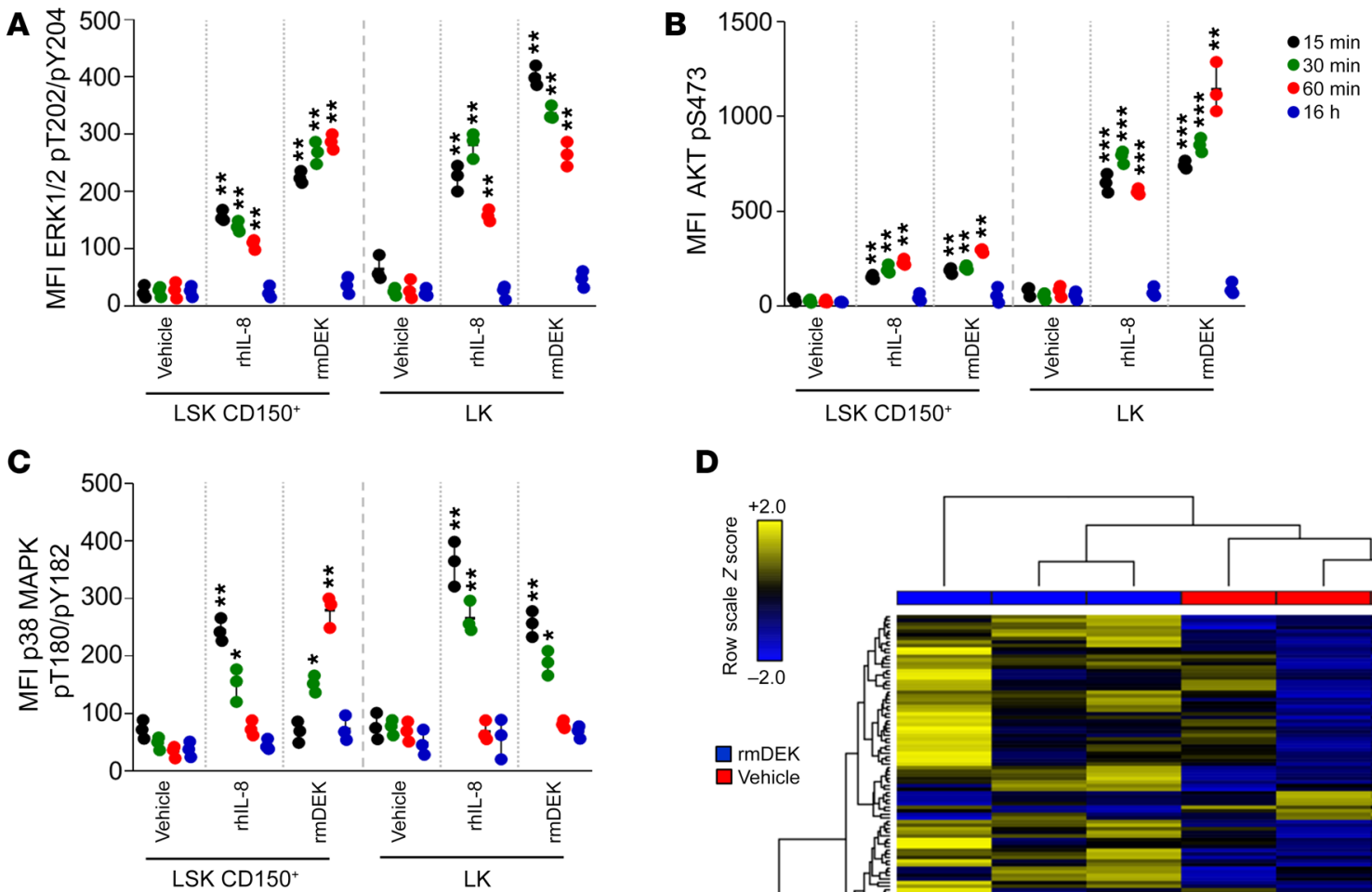

D

$\mathbf{E}$

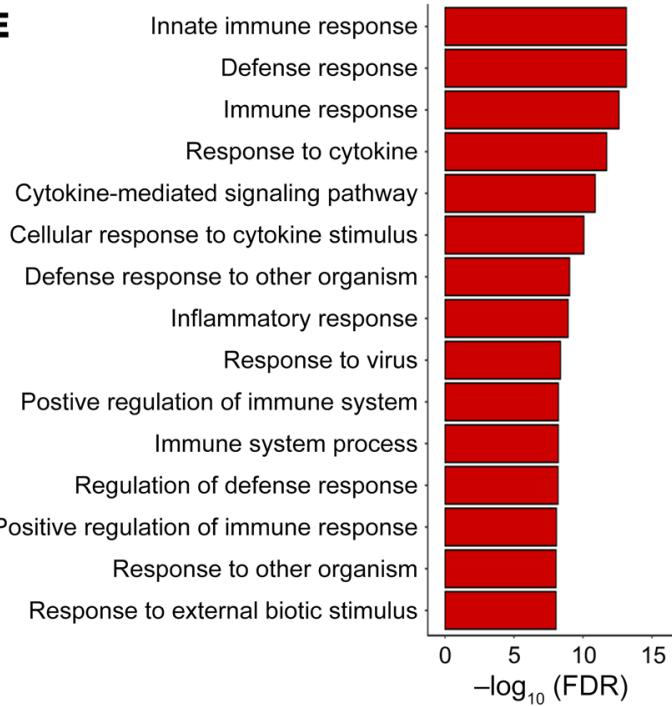

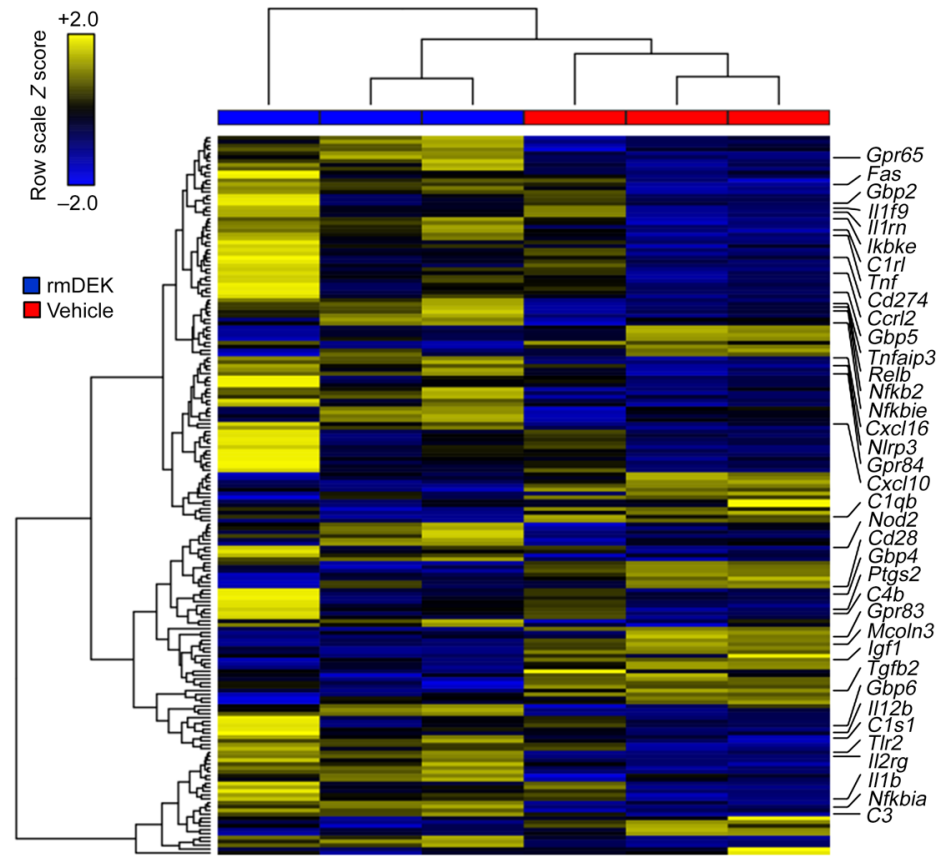

Figure 7. DEK stimulates ERK1/2, AKT, and p38 MAPK phosphorylation in LSK CD150+ and myeloid-enriched progenitor (LK) cells. (A-C) C57BL/6 BM LSK CD150+ cells and LK cells were examined by flow cytometry for ERK1/2 (A), AKT (B), and p38 MAPK (C) phosphorylation 15, 30 , and 60 minutes, or 16 hours following vehicle, rhIL-8, or rmDEK treatment. ${ }^{*} P<0.05,{ }^{* *} P<0.01$, and ${ }^{* * *} P<0.001$ when comparing groups with vehicle control at the same time point (1-way ANOVA with post hoc Tukey's multiple-comparisons test). Data are mean \pm SD of triplicate tubes. (D) Heatmap representation of the 186 differentially expressed genes (DEGs) when comparing vehicle-treated with rmDEK-treated Lin- BM. Unsupervised hierarchical clustering was performed to group samples (columns) and genes (rows) by similarities in data structure. Genes listed to the right of the heatmap represent factors with known induction by signaling cascades that exhibit crosstalk with CXCR2 response, including, but not limited to, AKT signaling, MAPK signaling, and NF- $\kappa B$ signaling. (E) Ontologic assessments conducted on DEK-mediated transcriptional changes with the RNA-Enrich program. A subset of concepts from the analysis ranked by significance is shown.

just rmDEK. WT rDEKs and the DEK mutants rhDBM-DEK and rhNLS-DEK equivalently enhanced ERK1/2 and AKT phosphorylation in LSK CD150+ cells (Supplemental Figure 7, G and H), demonstrating that extracellular DEK's nuclear function at the time points examined played no role in the ability of DEK to stimulate ERK1/2 or AKT phosphorylation.
To further confirm whether extracellular DEK is working through a cytokine/chemokine signaling pathway, we determined which molecular pathways were activated in DEK-treated immature cells. We transcriptionally profiled DEK-treated Lin ${ }^{-} \mathrm{BM}$ from age- and sex-matched $D e k^{-1-}$ mice exposed to rmDEK or vehicle control in vitro. Principal component analysis clearly separated 
the treatment groups (Supplemental Figure 8A). We identified 186 genes undergoing transcriptional changes driven by DEK exposure (Supplemental Figure 8B), which we visualized as a heatmap after unsupervised hierarchical clustering (Figure 7D). Numerous inflammatory cytokines were upregulated, including Tnf, Il12b, and Cxcl10, suggesting that DEK acts as a proinflammatory factor on Lin $^{-}$BM cells. Further suggesting a chemokine/cytokine-like role for DEK was the upregulation of a family of guanylate-binding proteins known to accentuate interferon signaling through interaction with $G$ protein-coupled receptors (GPCRs). Of note, signaling crosstalk between CXCR2 (a well-characterized GPCR) and a number of downstream effector pathways (AKT signaling, MAPK signaling, NF- $\kappa \mathrm{B}$ signaling) was identified from transcriptional profiling (Figure 7E, gene list). Furthermore, ontological assessment of DEK-mediated transcriptional changes revealed enrichment in innate immune system and cytokine response effectors (Figure 7E). Even though this analysis will not allow us to differentiate HSC/HPC populations from the rest of the $\mathrm{Lin}^{-} \mathrm{BM}$ cells, our RNA-Seq findings support our conclusion that rmDEK is most likely functioning through a cytokine/chemokine signaling pathway.

\section{Discussion}

We now demonstrate that extracellular DEK potently enhances cytokine-induced ex vivo expansion of mouse $\mathrm{BM}$ and human $\mathrm{CB}$ HSCs within 4 days. This is important, for if this is translated into clinical application with CB cells, it would be ideal to maximally enhance HSC expansion in the shortest amount of time possible (15). Moreover, exogenous DEK regulates hematopoiesis in vivo and in vitro in mice. It is striking that a protein normally found as a chromatin factor in the nucleus of cells can have another role as a soluble factor affecting hematopoiesis. To understand the mechanisms involved, we hypothesized that since DEK contained an ELR motif and acted as a chemoattractant for mature hematopoietic cells $(5,8)$, it likely functioned through the ELR motif-binding CXC chemokine receptor CXCR2. Blocking the ability of extracellular DEK to bind to CXCR2 with neutralizing CXCR2 antibody and examining the effects of DEK on HSC/HPC numbers in rmDEK-treated $\mathrm{Cxcr} 2^{-/-}$mice demonstrated that extracellular DEK required CXCR2 to mediate its effects on hematopoiesis in vitro and in vivo. rmDEK resulted in CXCR2-dependent phosphorylation of ERK1/2, AKT, and p38 MAPK, 3 well-characterized downstream CXCR2 signaling mediators. Collectively, these data provide strong evidence that DEK has cytokine/chemokine-like functions that can be leveraged for clinical applications.

DEK requires HSPGs to mediate regulatory effects on hematopoiesis. HSPGs can act as ligand-induced endocytic receptors (2931). Extracellular DEK requires HSPGs to become internalized by DEK-knockdown HeLa cells; pretreating cells with heparin blocked DEK uptake, and cells genetically modified to alter HSPG synthesis or positioning failed to take up DEK (12). DEK internalization by DEK-knockdown HeLa cells was associated with increased trimethylation of $\mathrm{H} 3 \mathrm{~K} 9$ in the nucleus, resulting in increased heterochromatin stability in vitro in human cells and in vivo in Drosophila $(2,12)$. We demonstrated that extracellular DEK requires HSPGs, endocytosis, and Gai protein signaling, but not CXCR2, for its ability to affect heterochromatin in $\mathrm{Dek}^{-/-}$LSK cells. However, DEK proteins lacking NLSs (rhNLS-DEK) or DNA-binding activity (rhDBM-DEK) were able to alter hematopoiesis in vivo and in vitro similarly to WT rDEK, suggesting that recombinant DEK's nuclear function is not responsible for DEK-mediated regulation of hematopoiesis in the context of our studies. What role, if any, DEKmediated heterochromatin remodeling in HSCs and HPCs may play remains to be determined. However, collectively our data suggest that DEK exerts its effect predominantly through a CXCR2 signaling pathway and not by mediating its nuclear function.

HSPGs play an important role in regulating chemokine function by binding to chemokines, protecting them against proteolysis, and sequestering chemokines on the cell surface, allowing for more efficient chemokine presentation and/or ligand-receptor clustering/signaling and thus lowering the activation threshold for optimal receptor signaling (30). Our data suggest that HSPGs serve as a coreceptor, not just an endocytosis receptor, for DEK, allowing for optimal DEK-mediated CXCR2-dependent cytokine regulation of hematopoiesis, as DEK-mediated regulation of hematopoiesis is CXCR2 dependent and DEK requires HSPGs for ERK1/2 and AKT phosphorylation.

A potential clinically relevant result is that extracellular DEK treatment greatly enhanced human and mouse HSC numbers in cytokine-stimulated ex vivo expansion assays, a process requiring CXCR2 and HSPGs. It remains unclear at present why treating C57BL/6 mice with rDEK in vivo resulted in increased phenotypically defined HSC numbers with decreased functional engraftment, while utilizing rDEK in ex vivo expansion assays enhanced engraftment of these cells. However, the finding reflects decreased CXCR4 expression on these cells and reduced homing efficiency with in vivo rDEK administration. We hypothesize that when giving rmDEK in vivo we may be affecting other cell types not examined in vitro in this study. One possibility is that factors may be produced following in vivo DEK treatment that indirectly influence hematopoiesis, possibly by decreasing the expression of CXCR4 and the homing capabilities of these cells. Thus, in vivo infusion of DEK in a clinical setting may allow for negative, rather than positive, effects on hematopoiesis, although there are ex vivo means to enhance expression of CXCR4 and the homing of human HSCs that could potentially be applied $(23,32,33)$.

In summary, we now show that DEK has profound effects on hematopoiesis ex vivo and in vivo in its soluble form, and that these regulating effects are mediated by DEK not as a chromatin factor, but rather via its cytokine/chemokine-like activity. We have demonstrated for the first time to our knowledge that DEK signals through CXCR2, an effect crucial for its ability to modulate hematopoiesis in vitro, ex vivo, and in vivo. DEK is active when administered systemically to mice. Interestingly, CXCR2 and IL-8 are two of the most frequently overexpressed genes in cell populations initiating acute myeloid leukemia (AML) and myeloid dysplastic syndrome (MDS), including LT-HSCs, ST-HSCs, and myeloid HPCs (34-36), and high expression is associated with poor clinical outcomes (36). This suggests that the IL-8/CXCR2 pathway may be a novel therapeutic target in AML and MDS. As IL-8 mediates DEK secretion by macrophages and neutrophils (5) and DEK acts through CXCR2, it may also be a useful target for modulating progression of MDS and AML. This is especially so in the context of DPP4-mediated truncation of DEK and/or IL-8; truncation of each has the capacity to block the action of not only its own full- 
length form, but also that of the other. In addition, alterations to DEK expression levels in patient plasma samples in some cancers (e.g., head and neck squamous cell carcinoma) correlate with poor disease outcome (37). Thus, it might be interesting to examine in the future extracellular DEK concentrations in MDS/AML patients to determine whether changes to DEK concentrations in the plasma correlate with disease progression/outcome.

\section{Methods}

Production of recombinant DEK. rmDEK and rhDEK were purified from insect cells as described previously $(13,38)$. Three days after infection with a high-titer virus stock, HighFive cells (Invitrogen) were harvested and washed 3 times with PBS prior to lysis with $2 \mathrm{ml}$ lysis buffer per $175-\mathrm{cm}^{2}$ flask (100 mM Tris-Cl [pH 7.5], $150 \mathrm{mM} \mathrm{NaCl}, 5 \mathrm{mM} \mathrm{MgCl}_{2}$, $1 \% \mathrm{NP}-40,5 \mathrm{mM}$ imidazole). The lysate was further treated with $1.3 \mathrm{M}$ $\mathrm{NaCl}$ for 20 minutes at room temperature, cleared $(100,000 \mathrm{~g}, 10$ minutes), and diluted with lysis buffer to a final concentration of 700 $\mathrm{mM} \mathrm{NaCl}$. After incubation for 1 hour at $4^{\circ} \mathrm{C}$ with Ni-nitrilotriacetic acid-agarose beads (QIAGEN), the beads were washed 3 times with 10 volumes of buffer 1 (50 mM Tris- $\mathrm{Cl}$ [pH 7.5], $150 \mathrm{mM} \mathrm{NaCl}, 50$ $\mathrm{mM}$ imidazole), 3 times with 10 volumes of buffer 2 (50 mM Tris-Cl [pH 7.5], $300 \mathrm{mM} \mathrm{NaCl}$, and $50 \mathrm{mM}$ imidazole), and again with 10 volumes of buffer 1 . Elution was performed with $50 \mathrm{mM}$ Tris- $\mathrm{Cl}(\mathrm{pH}$ 7.5) $/ 150 \mathrm{mM} \mathrm{NaCl} / 500 \mathrm{mM}$ imidazole. All recombinant proteins were dialyzed prior to being aliquoted and then stored at $-80^{\circ} \mathrm{C}$. Mutant DEK proteins were made as previously described $(13,38-41)$. See Supplemental Methods for further information.

Mice. Male and female C57BL/6J, Boy/J, B6×Boy/J F (herein referred to as $F_{1}$ ), and immunodeficient NSG (NOD.Cg-Prkdc $c^{\text {scid }}$ $\left.\mathrm{IL} \mathrm{rg}^{\mathrm{tm} \mathrm{wWj}^{\mathrm{j}}} / \mathrm{SzJ}\right)$ mice (8-10 weeks old) were obtained from an on-site breeding core facility at Indiana University School of Medicine. Male and female 8- to 10-week-old B6.129S2(C)-Cxcr $2^{\text {tmIMwm }} / \mathrm{J}$ homozy-

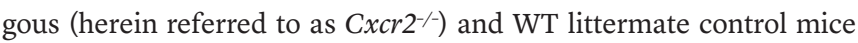
were bred for us by the Jackson Laboratory in a gnotobiotic facility. Male and female C57BL/6/129/SvEv (herein referred to as $\mathrm{Dek}^{-1-}$ ) mice were obtained from Gerard Grosveld, St. Jude Children's Hospital, Memphis, Tennessee, USA, and were adapted at the University of Michigan to a C57BL/6 background by back-breeding the homozygous knockout mouse with WT C57BL/6 mice for 9 generations $(8,13,42) . D^{-/-}$and littermate control mice were initially housed at the Animal Maintenance Facility at the University of Michigan Medical Center and then transferred to the Indiana University School of Medicine animal facility at 10-13 weeks of age. Animals were maintained under temperature- and light-controlled conditions $\left(21-24^{\circ} \mathrm{C}\right.$, 12-hour light/12-hour dark cycle) and were group-housed according to age, sex, and genotype. Mice were fed ad libitum. For all experiments, mice were matched by age and sex. For in vivo treatment with rmDEK, WT, Dek ${ }^{--}$, and Cxcr2 ${ }^{-/-}$mice were injected with $10 \mu \mathrm{g}$ dialyzed WT rmDEK, drmDEK, rhNLS-DEK, rhDBM-DEK, or vehicle control s.c. once a day for 2 days. BM was then collected at the indicated time points $(24,48,72,96$, or 144 hours after final injection) and analyzed.

Isolation of mouse Lin ${ }^{-}$BM cells and human CD $34^{+}$umbilical CB cells. Mouse BM was isolated from the femur of the indicated mouse strain immediately prior to use by flushing either in PBS (for transplantation studies; Lonza) or in Iscove's Modified Dulbecco's Medium (IMDM; Lonza) with HEPES, L-glutamine, and 10\% FBS (Fisher Scientific) for all culture experiments. Lineage depletion of mouse BM was per- formed using a mouse lineage cell depletion kit following the manufacturer's protocol (Miltenyi Biotec) using 2 sequential columns. Human umbilical CB was obtained from Cord:Use Cord Blood Bank (Orlando, Florida, USA). Upon arrival, CB was washed in PBS prior to FicollPaque PLUS (GE Healthcare Bio-Sciences AB) separation of mononuclear cells. The $\mathrm{CD} 34^{+} \mathrm{CB}$ cells were then isolated using a CD34 immunomagnetic selection kit according to the manufacturer's protocol (Miltenyi Biotec) using two sequential columns. The purity of mouse Lin- BM cells and human CD34+ CB cells was between $92 \%$ and $96 \%$.

Pretreatment of $B M$ cells with inhibitors and neutralizing agents. To examine the importance of Gai protein-coupled receptor signaling in DEK-mediated regulation of hematopoiesis, we incubated BM cells from the indicated mouse strains with 500 or $1000 \mathrm{ng} / \mathrm{ml} \mathrm{PT}$ (Sigma-Aldrich; catalog P7208) for 4 hours at $37^{\circ} \mathrm{C}$ immediately prior to utilizing them in experiments. To examine the role of HSPGs in DEK-mediated regulation of hematopoiesis, we utilized 3 different inhibitors: heparin (blocks binding of molecules to HSPGs), Xyl-Phe$\mathrm{NO}_{2}$ (disrupts proteoglycan biosynthesis), and heparinase III (cleaves HSPGs from cell surface). For heparin, BM cells were pretreated with $5 \mu \mathrm{g} / \mathrm{ml}$ heparin (from porcine intestinal mucosa; Sigma-Aldrich, catalog $\mathrm{H} 3393$ ) for 30 minutes at $37^{\circ} \mathrm{C}$ immediately prior to use in experiments and then washed out. For Xyl-PheNO${ }_{2}, \mathrm{BM}$ cells were treated with $100 \mu \mathrm{M}$ Xyl-PheNO ${ }_{2}$ (EMD Millipore, catalog 487870) in culture media (type determined by the experiment) 16 hours at $37^{\circ} \mathrm{C}$ immediately prior to use in experiments and then washed out. For heparinase III, BM cells were treated with $0.5 \mathrm{mIU} / \mathrm{ml}$ heparinase III (from Flavobacterium heparinum; Sigma-Aldrich, catalog H8891) in culture media (type determined by the experiment) for 4 hours at $37^{\circ} \mathrm{C}$ immediately prior to use in experiments and then washed out. To neutralize CXCR2 and CXCR4 on the cell surface, BM cells were incubated with $2.5 \mu \mathrm{g} / 10^{6}$ cells of anti-mouse CXCR2 purified rat monoclonal $\operatorname{IgG}_{2 \mathrm{~A}}$ antibody (R\&D Systems, clone 242216), anti-mouse CXCR4 purified rat monoclonal $\mathrm{IgG}_{2 \mathrm{~B}}$ antibody ( $\mathrm{R} \& \mathrm{D}$ Systems, clone 247506), or isotype rat IgG control (azide-free; R\&D Systems, catalog 6-001-F) for 30 minutes at room temperature immediately prior to use in experiments and then washed out. To examine whether DEK required endocytosis to mediate H3K9 trimethylation, we utilized the clathrindependent and -independent endocytosis inhibitor Pitstop 2, adding $30 \mu \mathrm{M}$ Pitstop 2 (Abcam Biochemicals) to the BM cells for 15 minutes at $37^{\circ} \mathrm{C}$ and then washing it out immediately prior to cells being incubated with rmDEK. To examine whether DEK functions differently in actively cycling cells, BM cells were incubated with $100 \mu \mathrm{g} / \mathrm{ml}$ hydroxyurea (Sigma-Aldrich, catalog H8627) for 45 minutes at $37^{\circ} \mathrm{C}$ and then washed twice immediately prior to use in experiments. Alternatively, high-specific-activity ${ }^{3} \mathrm{HTdr}$ kill assays were performed as previously described (23). Briefly, BM cells were treated with $50 \mu \mathrm{Ci}$ high-specific-activity ${ }^{3} \mathrm{HTdr}\left(20 \mathrm{Ci} / \mathrm{mmol}\right.$; DuPont NEN) at $37^{\circ} \mathrm{C}$ for 20 minutes or at room temperature for 40 minutes, then washed twice immediately prior to use in experiments.

Pretreatment of DEK with DEK-targeting aptamers. Anti-DEK aptamers were generated using SELEX technology as previously reported (8), and 2 single-stranded DNA aptamers with high affinity for rDEK proteins were selected: DTA-64 and DTA-85+ends. The library of single-stranded DNAs originally screened to obtain the DEK-targeted aptamers was used as a control. Recombinant DEK was pretreated with $100 \mathrm{ng} / \mathrm{ml}$ DTA-64, DTA-85+ends, aptamer library, or vehicle control for 1 hour at $37^{\circ} \mathrm{C}$. 
DPP4 truncation of rmDEK and rhCXCL8. Soluble human and porcine DPP4, prepared from human placental tissue or porcine kidney, were purchased from MP Biomedicals or Sigma-Aldrich (no. D7052), respectively, and used at approximately $0.25 \mu \mathrm{g}$ for every $1 \mu \mathrm{g}$ of fulllength rmDEK or rhIL-8 (also known as CXCL8; R\&D Systems, catalog 208-IL) protein per digestion at $37^{\circ} \mathrm{C}$ for at least 18 hours. Prior to use, truncated protein was treated with $5 \mathrm{nM}$ of the DPP4 inhibitor diprotin A (Enzo Life Science, catalog ALX-260-036) for 30 minutes at room temperature.

HPC assays. For HPC assays performed in agar, BM cells flushed from the femurs of the indicated mouse strain were plated at $5 \times 10^{4}$ cells $/ \mathrm{ml}$ in $0.3 \%$ semisolid agar medium and IMDM (Lonza) with $10 \%$ FBS (Fisher Scientific) that did or did not contain $10 \mathrm{ng} / \mathrm{ml} \mathrm{rmGM-CSF}$ (R\&D Systems, catalog 415-ML), $50 \mathrm{ng} / \mathrm{ml} \mathrm{rmSCF}$ (R\&D Systems, catalog 455-MC), 0.1-50 nM rmDEK, 0.1-50 nM GST rhDEK, 0.1-50 nM GST rhDBM-DEK, 0.1-50 nM GST rhNLS-DEK, 0.1-100 ng/ml rhIL-8 (R\&D Systems), 0.1-100 ng/ml rmMIP2 (also known as CXCL2; R\&D Systems, catalog 452-M2), $100 \mathrm{ng} / \mathrm{ml} \mathrm{rmMIP1 \alpha}$ (CCL3; R\&D Systems, catalog 450-MA), and/or $100 \mathrm{ng} / \mathrm{ml} \mathrm{rm6Ckine} \mathrm{(CCL21;} \mathrm{R \& D} \mathrm{Sys-}$ tems, catalog 457-6C), as indicated. Colonies were scored after 6 days of incubation at $5 \% \mathrm{CO}_{2}$ and lowered, $5 \% \mathrm{O}_{2}$ in a humidified chamber. For HPC assays performed in methylcellulose, BM cells flushed from the femurs of the indicated mouse strain were plated at $5 \times 10^{4}$ cells/ $\mathrm{ml}$ in $1 \%$ methylcellulose culture medium with $0.1 \mathrm{mM}$ hemin (SigmaAldrich), 30\% FBS, $1 \mathrm{U} / \mathrm{ml}$ recombinant human erythropoietin (rhEPO; Amgen), $50 \mathrm{ng} / \mathrm{ml} \mathrm{rmSCF}$, and $5 \% \mathrm{vol} / \mathrm{vol}$ pokeweed mitogen mouse spleen cell conditioned medium. Colonies were scored after 6 days of incubation at $5 \% \mathrm{CO}_{2}$ and lowered, $5 \% \mathrm{O}_{2}$ in a humidified chamber, and CFU-GM, BFU-E, and CFU-GEMM progenitors were distinguished by examining the morphology of the colonies. Where indicated, total number of colonies per femur was calculated (23).

Flow cytometry. For analyzing HSC and HPC phenotypes in mouse $\mathrm{BM}$ and human $\mathrm{CB}$, cells were collected at a concentration of approximately $2 \times 10^{6}$ cells per tube, washed in PBS, incubated in fluorescently conjugated anti-mouse or -human antibody cocktail for 20 minutes at room temperature, washed in PBS, and then fixed in $1 \%$ formaldehyde. The samples were analyzed on an LSR II flow cytometer (BD Biosciences). One microgram of antibody was used per one million cells. Single-color compensation and isotype controls were included for each experiment. Data analysis was performed using FlowJo 7.6.3 software (Tree Star). Gates were determined using fluorescence minus one controls. The percent of each population was used to calculate the absolute number of each population per femur - for mouse phenotyping markers: FITC- or Pacific Blue-mouse lineage cocktail (CD3, Gr-1, CD11b, CD45R, Ter119; BioLegend, catalog 133302 and 133310), PE-CF594-anti-Ly6A/E (also known as Sca-1; clone D7; BD Biosciences), APC-H7-anti-CD117 (c-Kit; clone 2B8; BD Biosciences), APC- or PE-anti-CD135 (Flt3; clone A2F10.1; BD Biosciences), PE- or BV421-anti-CD34 (clone RAM34; BD Biosciences), PerCP-Cy5.5-anti-CD16/CD32 (Fc $\gamma$ R; clone 2.4G2; BD Biosciences), PE- or BV421-anti-CD150 (clone Q38-480; BD Biosciences), FITCor BV421-anti-CD48 (clone HM48-1; BD Biosciences), FITC- or BV421-anti-CD41 (clone MWReg30; BD Biosciences), FITC-antiCD45.1 (clone A20; BD Biosciences), APC-anti-CD45.2 (clone 104; BD Biosciences), and APC-anti-CD184 (clone 2B11/CXCR4; BD Biosciences); for human phenotyping markers: FITC-anti-CD34 (clone 581; BD Biosciences), PE-anti-CD38 (clone HIT2; BD Biosciences),
PE-CF594-anti-CD45RA (clone HI100; BD Biosciences), PE-Cy7anti-CD90 (clone 5E10; BD Biosciences), PerCP-Cy5.5-anti-CD49f (clone GoH3; BD Biosciences), and APC-anti-CD45 (clone HI30; BD Biosciences). HSC and HPC populations for mice were defined as follows - LSK cells: Lin ${ }^{-}$Sca-1 ${ }^{+}{ }^{+}$-Kit ${ }^{+}$; LT-HSCs: LSK Flt3 ${ }^{-}$CD $34^{-}$or LSK Flt3 ${ }^{-} \mathrm{CD} 34^{-} \mathrm{CD} 150^{+} \mathrm{CD} 48^{-} \mathrm{CD} 41^{-}$; ST-HSCs: LSK Flt3-CD34+' MPPs: LSK Flt $3{ }^{+} \mathrm{CD}^{2} 4^{+}$. HSCs in human $\mathrm{CB}$ were defined as $\mathrm{Lin}^{-} \mathrm{CD} 34^{+} \mathrm{CD} 38^{-}$ $\mathrm{CD}^{2} 5 \mathrm{RA}^{-} \mathrm{CD} 90^{+} \mathrm{CD}_{49 \mathrm{f}^{+}}$(43). For analysis of transplantation experiments, APC-anti-CD45.2 (clone 104) and FITC-anti-CD45.1 (clone A20) were purchased from BD Biosciences. For all antibodies used in these studies, the validation for the relevant species and applications can be found on the indicated manufacturer's website.

RNA-Seq library preparation and analysis. BM from $\mathrm{Dek}^{-/-}$mice was cultured in RPMI-1640 media (Lonza) with 10\% FBS (Fisher Scientific) for 16 hours with $50 \mathrm{nM}$ rmDEK or vehicle control. The AllPrep DNA/RNA Mini Kit (QIAGEN) was utilized to isolate RNA from cell lysates. RNase-Free DNase (QIAGEN) was used to eliminate contaminating genomic DNA. RNA integrity was evaluated using an Agilent TapeStation. Strand-specific libraries for 3 replicate pairs of DEK- and vehicle-treated samples were prepared using the TruSeq Stranded mRNA kit (Illumina) according to the manufacturer's protocol. Library preparations were done in conjunction with the University of Michigan Sequencing Core. Single-end, strand-specific libraries were sequenced with an Illumina HiSeq 4000 (50-nucleotide read length). Sequencing results were run through a computational pipeline to trim (Trim Galore; https://www.bioinformatics.babraham.ac.uk/projects/trim_galore/) low-quality bases and adapters, align reads (STAR; https://code. google.com/archive/p/rna-star/) to the mouse reference genome (UCSC mm10 from iGenomes; Illumina), assemble aligned reads (HTseq) (44), and call differentially expressed genes (using the quasilikelihood method with a paired design in edgeR) (45). An absolute fold change $(\mathrm{FC})>1.5$ and FDR $<0.05$ were required in order for a gene to be considered differentially expressed. Pathway analysis to identify enriched Gene Ontology (GO) terms was conducted using RNA-Enrich (46). The data discussed in this publication have been deposited in the NCBI's Gene Expression Omnibus (GEO GSE126875).

Intracellular phosphoprotein staining. Mouse $\mathrm{Lin}^{-} \mathrm{BM}$ cells were left unstimulated or stimulated with $100 \mathrm{ng} / \mathrm{ml} \mathrm{rmSCF}$ (R\&D Systems), $100 \mathrm{ng} / \mathrm{ml}$ rhSDF1 $\alpha$ (also known as CXCL12; R\&D Systems, catalog 350-NS), $100 \mathrm{ng} / \mathrm{ml} \mathrm{rhIL-8} \mathrm{(R \& D} \mathrm{Systems),} 50 \mathrm{nM} \mathrm{rmDEK}$, $50 \mathrm{nM}$ GST WT rhDEK, $50 \mathrm{nM}$ GST rhDBM-DEK, or $50 \mathrm{nM}$ GST rhNLS-DEK for 15 minutes, 30 minutes, 60 minutes, or 16 hours at $37^{\circ} \mathrm{C}$. Cells were put on ice, stained for surface markers, fixed with BD Cytofix Buffer (BD Biosciences) for 10 minutes at $37^{\circ} \mathrm{C}$, and then permeabilized with BD Phosflow Perm Buffer III (BD Biosciences) for 30 minutes on ice. Cells were then washed twice, stained with antibody against the indicated phosphoprotein, and then analyzed on an LSR II flow cytometer. The manufacturer-recommended (BD Biosciences) concentration of antibody was used. Data analysis was performed using FlowJo 7.6.3 software. For intracellular phosphoprotein staining, the following antibodies were used: PE-anti-ERK1/2 pT202/ p204 (clone 20A; BD Biosciences), PE-anti-AKT pS473 (clone M8961; BD Biosciences), and PE-anti-p38 MAPK pT180/pY182 (clone 36/ p38 pT180/pY182; BD Biosciences).

HSC expansion assays. For expansion of human HSCs, CD $34^{+} \mathrm{CB}$ cells were isolated as described above, then cultured at 50,000 cells/ well in RPMI-1640 media (Lonza) with 10\% FBS (Fisher Scientific), 
$100 \mathrm{ng} / \mathrm{ml} \mathrm{rhSCF}$ (R\&D Systems, catalog 7466-SC-010/CF), 100 $\mathrm{ng} / \mathrm{ml}$ rhTPO (R\&D Systems, catalog 288-TP-200/CF), $100 \mathrm{ng} / \mathrm{ml}$ rhFlt3L (BioLegend, catalog 710802), and either $50 \mathrm{nM}$ rhDEK or vehicle control at $37^{\circ} \mathrm{C}$ at $5 \% \mathrm{CO}_{2}$ and lowered, $5 \% \mathrm{O}_{2}$ in a humidified chamber. Cells were removed after 4 days and counted. Flow cytometry was then performed, examining HSC frequency in culture at time of input and at 4 days of culture. The percent HSCs and number of cells per well were then used to calculate HSC expansion. For expansion of mouse LT-HSCs, Lin- BM cells were isolated as described above, then cultured at 50,000 cells/well in RPMI-1640 media (Lonza) with 10\% FBS (Fisher Scientific), $100 \mathrm{ng} / \mathrm{ml} \mathrm{rmSCF} \mathrm{(R \& D} \mathrm{Systems,} \mathrm{catalog}$ 455-MC-010), $100 \mathrm{ng} / \mathrm{ml} \mathrm{rmTPO} \mathrm{(R \& D} \mathrm{Systems,} \mathrm{catalog} \mathrm{488-TO-}$ 055/CF), $100 \mathrm{ng} / \mathrm{ml} \mathrm{rmFlt3L} \mathrm{(BioLegend,} \mathrm{catalog} \mathrm{550706),} \mathrm{and} 50$ nM rmDEK, 50 nM GST rhDEK, 50 nM GST rhNLS-DEK, 50 nM GST rhDBM-DEK, or vehicle control at $37^{\circ} \mathrm{C}$ at $5 \% \mathrm{CO}_{2}$ and lowered, $5 \%$ $\mathrm{O}_{2}$ in a humidified chamber. The mouse LT-HSC expansion assay was then performed similarly to the human assay.

HSC and HPC engrafting studies. To analyze the effect of rmDEK treatment in vivo, C57BL/6 mice were injected with $10 \mu \mathrm{g}$ dialyzed WT rmDEK, drmDEK, or vehicle control s.c. once a day for 2 days. BM was then collected 24 or 48 hours after final injection. Competitive transplantations were performed using donor BM cells from vehicle- or rmDEK-treated C57BL/6 mice (CD45.1-CD45.2 ${ }^{+}$). These cells were mixed at a 1:1 ratio $\left(1 \times 10^{5}\right.$ cells $)$ with competitor $\mathrm{BM}$ from Boy/J mice $\left(\mathrm{CD} 45.1^{+} \mathrm{CD} 45.2^{-}\right)$and injected intravenously into $\mathrm{B} 6 \times \mathrm{Boy} / \mathrm{J} \mathrm{F}_{1}$ mice $\left(\mathrm{CD} 45.1^{+} \mathrm{CD} 45.2^{+}\right)$that had been lethally irradiated (950 cGy) 24 hours prior to transplantation. Following 1, 2, 4, and 6 months, the percentage of donor $\mathrm{CD} 45.1^{-} \mathrm{CD} 45.2^{+}$cells in the PB was determined by flow cytometry.

Homing studies. C57BL/6 mice (CD45.1 $\left.{ }^{-} \mathrm{CD} 45.2^{+}\right)$were injected with $10 \mu$ g dialyzed WT rmDEK or vehicle control s.c. once a day for 2 days. BM was then collected 24 or 48 hours after final injection. Twelve million cells from these mice were then injected i.v. into lethally irradiated (950 cGy, 24 hours prior) $\mathrm{B} 6 \times \mathrm{Boy} / \mathrm{J} \mathrm{F}_{1}$ mice $\left(\mathrm{CD} 45.1^{+} \mathrm{CD} 45.2^{+}\right.$) hosts. Eighteen hours following injection, BM from host mice was collected and analyzed by flow cytometry for the presence of CD $45.1^{-}$ CD 45.2 $2^{+}$LSK CD150 ${ }^{+}$, LSK, and LK cells.

Limiting dilution analysis. For mouse studies, progeny cells from C57BL/6 (CD45.1-CD45.2 ${ }^{+}$) mouse Lin $^{-}$BM HSC ex vivo expansion assays were utilized. Increasing doses of uncultured or 4-day-cultured vehicle- or rmDEK-treated mouse Lin' cells $(10,000,25,000$, or 100,000 cells or the progeny of the equivalent number of cells) were mixed with $1 \times 10^{5}$ BoyJ $\left(\mathrm{CD} 45.1^{+} \mathrm{CD} 45.2^{-}\right) \mathrm{BM}$ competitor cells, then intravenously injected into $\mathrm{F}_{1}$ mice $\left(\mathrm{CD} 45.1^{+} \mathrm{CD} 45.2^{+}\right)$that had been lethally irradiated ( 950 cGy) 24 hours before transplantation. Following 2 and 4 months, the percentage of donor CD $45.1^{-} \mathrm{CD} 45.2^{+}$ cells in the PB and BM (month 4 only) was determined by flow cytometry. The number of mice with greater than $15 \%$ donor-derived BM cells $\left(\mathrm{CD} 45.1^{-} \mathrm{CD} 45.2^{+}\right)$was determined for each dose. The HSC frequency was calculated using L-Calc software (STEMCELL Technologies) and plotted using ELDA software (http://bioinf.wehi.edu.au/ software/elda/). For secondary transplants, $2 \times 10^{6} \mathrm{BM}$ cells from the above primary recipient mice were intravenously injected into lethally irradiated $\mathrm{F}_{1}$ mice. The percentage of $\mathrm{CD} 45.1^{-} \mathrm{CD} 45.2^{+}$cells in the $\mathrm{PB}$ and $\mathrm{BM}$ (month 4 only) was evaluated. For human studies, the progeny cells from human $\mathrm{CD} 34^{+} \mathrm{CB}$ HSC ex vivo expansion assays were utilized. Increasing doses of uncultured or 4-day-cultured vehicle- or rhDEK-treated human CD34 ${ }^{+} \mathrm{CB}$ cells $(500,2500$, or 10,000 cells or the progeny of the equivalent number of cells) were injected into NSG mice that had been sublethally irradiated ( $350 \mathrm{cGy}$ ) 24 hours before transplantation. Following 2 and 4 months, the percentage of donor human $\mathrm{CD} 45^{+}$cells in the PB and BM (month 4 only) was determined by flow cytometry. The number of mice with greater than $1 \%$ human donor-derived BM cells was determined for each dose. The HSC frequency was calculated using L-Calc software and plotted using ELDA software. For secondary transplants, $2 \times 10^{6} \mathrm{BM}$ cells from the above primary recipient mice were intravenously injected into sublethally irradiated NSG mice. The percentage of human $\mathrm{CD} 45^{+}$cells in the PB and BM (month 4 only) was evaluated.

ImageStream analysis of $\mathrm{H} 3 \mathrm{~K} 9$ trimethylation. $\mathrm{Lin}^{-} \mathrm{BM}$ cells from $D e k^{-/}$and littermate WT control mice were stained for the indicated surface markers, then fixed/permeabilized using BD Cytofix/Cytoperm Fixation/Permeabilization Kit (BD Biosciences) according to the manufacturer's instructions. Cells were then incubated with a 1:200 dilution of anti-trimethyl histone H3 (Lys9) Alexa Flour 488 antibody (EMD Millipore Corporation, catalog 07-442-AF488) for 30 minutes at $4^{\circ} \mathrm{C}$ in the dark. Following staining, cells were washed and refixed using BD Cytofix buffer for 10 minutes. Cells were then washed, resuspended in $20 \mu \mathrm{M}$ DRAQ5 (BD Biosciences, catalog 564903), incubated for 10 minutes at room temperature, and then analyzed. 50,000$80,000 \mathrm{Lin}^{-}, \mathrm{c}-\mathrm{Kit}^{+}$events were collected using the slow stream setting at a $\times 40$ objective for all samples on an ImageStreamX MKII (Amnis/ EMD Millipore) using INSPIRE ImageStreamX MKII Software and analyzed using IDEAS Image Data Exploration and Analysis Software version 4 (Amnis/EMD Millipore). Spectral compensation was digitally performed on a pixel-by-pixel basis prior to data analysis. Cells that were in focus when imaged, then single, live cells were gated based on their aspect ratio and area of the bright-field image. Samples were then gated on the LSK population, and the relationship between the H3K9me3 and DRAQ5 staining was measured using the "similarity" feature in the IDEAS software. The similarity score, which is the log-transformed Pearson's correlation coefficient between the pixel values of 2 image pairs, provided a measure of the degree of nuclear staining of H3K9me3 by comparing H3K9me3 staining with DRAQ5 staining by measuring the pixel intensity correlation between the 2 images. Cells with high similarity scores demonstrated greater H3K9 trimethylation in the nucleus, and cells with lower similarity scores had less H3K9 trimethylation in the nucleus. Gating of the similarity score histograms based on LSK cells from untreated littermate control BM was used to calculate the percent change in $\mathrm{H} 3 \mathrm{~K} 9 \mathrm{me} 3$ in the nuclei of littermate control and $\mathrm{Dek}^{-/-} \mathrm{BM}$ cells following different treatments.

Statistics. Results are expressed as mean values \pm SD or SEM as indicated. Two-tailed Student's $t$ test was used where indicated. Oneway ANOVA with post hoc Tukey's multiple-comparisons test was used when comparing 3 or more groups. Poisson statistical analysis was used for the limiting dilution assays. A $P$ value less than 0.05 was considered statistically significant.

Study approval. The Indiana University Institutional Review Board approved all CB studies. All animal procedures were approved by the University of Michigan and Indiana University Committees on Use and Care of Animals.

\section{Author contributions}

MLC, NMV, DMM, and HEB conceived the research, designed and performed experiments, interpreted data, and wrote the 
manuscript. ML, SC, AKS, HG, RCG, XH, MAS, CTL, and FK performed experiments and produced recombinant proteins.

\section{Acknowledgments}

These studies were supported by US Public Health Service grants from the NIH to HEB: R01 DK 109188, U54 DK 106846, R01 HL 056416, R01 HL 112669, and R35 HL 139599. Additional grant support to HEB was provided by the Indiana Clinical and Translational Sciences Institute (CTSI) core pilot grant program. Grant support to MLC was provided by Indiana University Simon Cancer Center American Cancer Society Institutional Research Pilot Grant IRG-16-192-31. MLC was also supported by NIH training grant DK 007519 to HEB for some of these studies. AKS was funded by the Cancer Biology Program of the University of Michigan and grant F30-CA-210379 from the NIH. DMM, NMV, CL, and MS were supported by R01 DK 109188 from the NIH, and DMM and NMV were additionally funded by a grant from the Rheuma- tology Research Foundation. Grant support for HG and FK was provided by the China Scholarship Council, Deutsche Forschungsgemeinschaft (DFG KA 2799/1), and the START Program of the Faculty of Medicine, RWTH Aachen University.

Address correspondence to: Hal E. Broxmeyer, Department of Microbiology and Immunology, Indiana University School of Medicine, 950 West Walnut Street, R2-302, Indianapolis, Indiana 46202, USA. Phone: 317.274.7510; Email: hbroxmey@ iupui.edu. Or to: David M. Markovitz, Department of Internal Medicine, University of Michigan Medical Center, 1150 W. Medical Center Drive, Ann Arbor, Michigan 48109, USA. Phone: 734.647.1786; Email: dmarkov@umich.edu. Or to: Maegan Capitano, Department of Microbiology and Immunology, Indiana University School of Medicine, 950 West Walnut Street, R2-302, Indianapolis, Indiana 46202, USA. Phone: 317.274.7555; Email: malcapit@iupui.edu.
1. Capitano ML, Broxmeyer HE. A role for intracellular and extracellular DEK in regulating hematopoiesis. Curr Opin Hematol. 2017;24(4):300-306.

2. Kappes F, et al. The DEK oncoprotein is a Su(var) that is essential to heterochromatin integrity. Genes Dev. 2011;25(7):673-678.

3. Waldmann T, Baack M, Richter N, Gruss C. Structure-specific binding of the proto-oncogene protein DEK to DNA. Nucleic Acids Res. 2003;31(23):7003-7010.

4. Mor-Vaknin N, et al. DEK in the synovium of patients with juvenile idiopathic arthritis: characterization of DEK antibodies and posttranslational modification of the DEK autoantigen. Arthritis Rheum. 2011;63(2):556-567.

5. Mor-Vaknin N, et al. The DEK nuclear autoantigen is a secreted chemotactic factor. Mol Cell Biol. 2006;26(24):9484-9496.

6. Kappes F, et al. DEK is a poly(ADP-ribose) acceptor in apoptosis and mediates resistance to genotoxic stress. Mol Cell Biol. 2008;28(10):3245-3257.

7. Dong X, Michelis MA, Wang J, Bose R, DeLange T, Reeves WH. Autoantibodies to DEK oncoprotein in a patient with systemic lupus erythematosus and sarcoidosis. Arthritis Rheum. 1998;41(8):1505-1510.

8. Mor-Vaknin N, et al. DEK-targeting DNA aptamers as therapeutics for inflammatory arthritis. Nat Commun. 2017;8:14252.

9. Andersson U, et al. High mobility group 1 protein (HMG-1) stimulates proinflammatory cytokine synthesis in human monocytes. J Exp Med. 2000;192(4):565-570.

10. Lotze MT, Tracey KJ. High-mobility group box 1 protein (HMGB1): nuclear weapon in the immune arsenal. Nat Rev Immunol. 2005;5(4):331-342.

11. Magna M, Pisetsky DS. The role of HMGB1 in the pathogenesis of inflammatory and autoimmune diseases. Mol Med. 2014;20:138-146.

12. Saha AK, et al. Intercellular trafficking of the nuclear oncoprotein DEK. Proc Natl Acad Sci US A. 2013;110(17):6847-6852.

13. Broxmeyer HE, et al. DEK regulates hematopoietic stem engraftment and progenitor cell proliferation. Stem Cells Dev. 2012;21(9):1449-1454.
14. Broxmeyer HE, et al. Concise review: role of DEK in stem/progenitor cell biology. Stem Cells. 2013;31(8):1447-1453.

15. Broxmeyer HE. The history of cord blood transplantation/biology and prespective for future efforts to enhance the field. Cell Gene Ther Insights. 2017;3(7):521-530.

16. Broxmeyer HE, Farag S, Rocha V. In: Forman SJ, Negrin RS, Antin JH, Appelbaum FR, eds. Thomas' Hematopoietic Cell Transplantation. Oxford, United Kingdom: John Wiley \& Sons; 2016:437-455.

17. Strieter RM, et al. The functional role of the ELR motif in CXC chemokine-mediated angiogenesis. J Biol Chem. 1995;270(45):27348-27357.

18. Broxmeyer HE, et al. Human chemokines: enhancement of specific activity and effects in vitro on normal and leukemic progenitors and a factor-dependent cell line and in vivo in mice. Ann Hematol. 1995;71(5):235-246.

19. Broxmeyer HE, Kim CH. Regulation of hematopoiesis in a sea of chemokine family members with a plethora of redundant activities. Exp Hematol.1999;27(7):1113-1123.

20. Daly TJ, LaRosa GJ, Dolich S, Maione TE, Cooper S, Broxmeyer HE. High activity suppression of myeloid progenitor proliferation by chimeric mutants of interleukin 8 and platelet factor 4 . J Biol Chem. 1995;270(40):23282-23292.

21. Broxmeyer HE, Capitano M, Campbell TB, Hangoc G, Cooper S. Modulation of hematopoietic chemokine effects in vitro and in vivo by DPP-4/CD26. Stem Cells Dev. 2016;25(8):575-585

22. Broxmeyer HE, Cooper S, Cacalano G, Hague NL, Bailish E, Moore MW. Involvement of interleukin (IL) 8 receptor in negative regulation of myeloid progenitor cells in vivo: evidence from mice lacking the murine IL-8 receptor homologue. J Exp Med. 1996;184(5):1825-1832.

23. Broxmeyer HE, et al. Dipeptidylpeptidase 4 negatively regulates colony-stimulating factor activity and stress hematopoiesis. Nat Med. 2012;18(12):1786-1796.

24. Christopherson KW, Hangoc G, Broxmeyer HE. Cell surface peptidase CD26/dipeptidylpeptidase IV regulates CXCL12/stromal cell-derived factor-1 alpha-mediated chemotaxis of human cord blood CD34+ progenitor cells. J Immunol. 2002;169(12):7000-7008.

25. Ou X, O'Leary HA, Broxmeyer HE. Implications of DPP 4 modification of proteins that regulate stem/progenitor and more mature cell types. Blood. 2013;122(2):161-169.

26. Broxmeyer HE. Regulation of hematopoiesis by chemokine family members. Int J Hematol. 2001;74(1):9-17.

27. Dutta D, Williamson CD, Cole NB, Donaldson JG. Pitstop 2 is a potent inhibitor of clathrin-independent endocytosis. PLOS ONE. 2012;7(9):e45799.

28. Waugh DJ, Wilson C. The interleukin-8 pathway in cancer. Clin Cancer Res. 2008;14(21):6735-6741.

29. Belting M. Heparan sulfate proteoglycan as a plasma membrane carrier. Trends Biochem Sci. 2003;28(3):145-151.

30. Sarrazin S, Lamanna WC, Esko JD. Heparan sulfate proteoglycans. Cold Spring Harb Perspect Biol. 2011;3(7):a004952.

31. Williams KJ, Fuki IV. Cell-surface heparan sulfate proteoglycans: dynamic molecules mediating ligand catabolism. Curr Opin Lipidol. 1997;8(5):253-262.

32. Guo B, Huang X, Cooper S, Broxmeyer HE. Glucocorticoid hormone-induced chromatin remodeling enhances human hematopoietic stem cell homing and engraftment. Nat Med. 2017;23(4):424-428.

33. Huang X, Guo B, Liu S, Wan J, Broxmeyer HE. Neutralizing negative epigenetic regulation by HDAC5 enhances human haematopoietic stem cell homing and engraftment. Nat Commun. 2018;9(1):2741.

34. Cazzola M, Della Porta MG, Malcovati L. The genetic basis of myelodysplasia and its clinical relevance. Blood. 2013;122(25):4021-4034.

35. Heuser M, et al. Cell of origin in AML: susceptibility to MN1-induced transformation is regulated by the MEIS1/AbdB-like HOX protein complex. Cancer Cell. 2011;20(1):39-52.

36. Schinke C, et al. IL8-CXCR2 pathway inhibition as a therapeutic strategy against MDS and AML stem cells. Blood. 2015;125(20):3144-3152.

37. Wise-Draper T, et al. Decreased plasma DEK oncogene levels correlate with p16-negative dis- 
ease and advanced tumor stage in a case-control study of patients with head and neck squamous cell carcinoma. Transl Oncol. 2018;11(1):168-174.

38. Kappes F, Scholten I, Richter N, Gruss C, Waldmann T. Functional domains of the ubiquitous chromatin protein DEK. Mol Cell Biol. 2004;24(13):6000-6010.

39. Devany M, Kotharu NP, Matsuo H. Solution NMR structure of the C-terminal domain of the human protein DEK. Protein Sci. 2004;13(8):2252-2259.

40. Böhm F, et al. The SAF-box domain of chromatin protein DEK. Nucleic Acids Res.
2005;33(3):1101-1110.

41. Devany M, Kappes F, Chen KM, Markovitz DM, Matsuo H. Solution NMR structure of the N-terminal domain of the human DEK protein. Protein Sci. 2008;17(2):205-215.

42. Wise-Draper TM, et al. DEK proto-oncogene expression interferes with the normal epithelial differentiation program. Am J Pathol. 2009;174(1):71-81.

43. van Galen P, et al. The unfolded protein response governs integrity of the haematopoietic stem-cell pool during stress. Nature.
2014;510(7504):268-272.

44. Anders S, Pyl PT, Huber W. HTSeq - a Python framework to work with high-throughput sequencing data. Bioinformatics. 2015;31(2):166-169.

45. Lun AT, Chen Y, Smyth GK. It's DE-licious: a recipe for differential expression analyses of RNAseq experiments using quasi-likelihood methods in edgeR. Methods Mol Biol. 2016;1418:391-416.

46. Lee C, Patil S, Sartor MA. RNA-Enrich: a cut-off free functional enrichment testing method for RNA-seq with improved detection power. Bioinformatics. 2016;32(7):1100-1102. 